\title{
Goodness-of-fit test for multistable Lévy processes
}

\author{
R. Le Guével \\ Univ Rennes, CNRS, IRMAR - UMR 6625, F-35000 Rennes, France \\ ronan.leguevel@univ-rennes2.fr
}

\begin{abstract}
The multistable processes are extensions of stable processes, where the index of stability is replaced by a function ranging in $(0,2)$. The aim of this article is to build a statistical test which is able to detect a multistable behavior of a process belonging to the class of the multistable Lévy processes. The properties are stated for a discrete observation scheme of one trajectory.
\end{abstract}

Keywords: Multistable process, Lévy process, nonparametric test, energy test.

\section{Introduction}

Most of time, homogeneous processes are used for real datasets as first intention models. If one deals with processes with jumps, one can use a Lévy process (or a transformation of it) as a basic model. Various generalisations of this process have been introduced these last years in order to overcome the lack of flexilibility of these models. We can mention for instance the introduction of the Multifractional Brownian Motion (Peltier and Lévy-Véhel 1995; Benassi, Jaffard, and Roux 1997; Ayache and Lévy-Véhel 2000; Herbin 2006), or the Linear Multifractional Stable Motion (Stoev and Taqqu 2004, 2005) as models where the regularity of the observation is tuned by a function $H$, which is playing the role of the local Hurst index. For these models, one statistical challenge is to build a test in order to decide if the regularity $H$ is constant or not along the trajectory. One can see for instance (Biermé and Richard 2010; Richard and Vu 2019) where the authors deal with anisotropic multifractional Brownian fields. For processes with jumps, for the same reason it is interesting to deal with stochastic processes whose local Hölder exponent changes in a controlled manner, it is convenient to consider models where the jump intensity is allowed to vary in time.

Recall that a process is called $\alpha$-stable $(0<\alpha \leq 2)$ if all its finite-dimensional distributions are $\alpha$-stable (see Samorodnitsky and Taqqu 1994). A generalisation of these $\alpha$-stable processes has been introduced in Falconer and Lévy-Véhel (2008) in order to provide good models for data containing a distribution of discontinuities which is varying. They are useful models for financial records, EEG or natural terrains for instance. It is a practical way to deal with non-stationarities observed in various real-data phenomena, since a multistable process $X$ is tangent, at each time $u$, to a stable process $Z_{u}$ in the following sense (Falconer 2002, 2003):

$$
\lim _{r \rightarrow 0} \frac{X(u+r t)-X(u)}{r^{h(u)}}=Z_{u}(t)
$$


for a suitable $h(u)$ (the limit (1) is taken either in finite dimensional distributions or, when $X$ has a version with càdlàg paths, in distribution - one then speaks of strong localisability).

Some properties of these processes has already been studied. We may mention for instance the behaviour of their distribution (Ayache 2013), some regularity properties (Falconer and Liu 2012; Biermé and Lacaux 2013; Le Guével and Lévy-Véhel 2013; Le Guével 2018) or some semi-martingale representations (Le Guével, Lévy-Véhel, and Liu 2012). The multistable subordinator provides also good models for processes in heterogeneous environments. We may find some limit theorems for the subordinator and the multifractional Poisson processes in Molchanov and Ralchenko (2015). The study of its generator and some related differential equations for multistable Markov processes are also major subjects (see e.g. Beghin and Ricciuti 2018; Orsingher, Ricciuti, and Toaldo 2016; Ricciuti and Toaldo 2017).

The remainder of this paper is organized as follows: the next section is devoted to the definitions of two versions of the Lévy multistable process, using their Ferguson-Klass-LePage representation. In section 3, we introduce general notations. We specify some estimation tools and we state some auxiliary properties about these tools. Section 4 presents the problem of testing the multistability of a Lévy multistable model. We give in this section the main results of convergence of our testing procedure. Section 5 is dedicated to technical lemmas, useful for the proofs of the main theorems. Finally, we have gathered all the proofs in section 6 .

\section{Multistable processes}

For $\alpha \in(0,2]$, recall that the stochastic integral $I(f):=\int f(x) M(d x)$ of a real function $f$ with respect to $M$ exists if, for instance $M$ is a symmetric $\alpha$-stable random measure on $\mathbb{R}$, with the Lebesgue measure as the control measure, and if $f$ is measurable and satisfies $\int_{\mathbb{R}}|f(x)|^{\alpha} d x<+\infty$ (see Samorodnitsky and Taqqu 1994). Many symmetric stable processes $\{Y(t), t \in \mathbb{R}\}$ admit the stochastic integral representation

$$
Y(t)=\int f_{t}(x) M(d x)
$$

Write $S_{\alpha}(\sigma, \beta, \mu)$ for the $\alpha$-stable distribution with scale parameter $\sigma$, skewness $\beta$ and shift parameter $\mu$. The marginal distribution of $Y$ is therefore $Y(t) \sim S_{\alpha}\left(\sigma_{f_{t}}, 0,0\right)$ where $\sigma_{f_{t}}=$ $\left(\int_{\mathbb{R}}\left|f_{t}(x)\right|^{\alpha} d x\right)^{1 / \alpha}$.

We will restrict our attention to the standard symmetric $\alpha$-stable Lévy process on the interval $[0,1]$, which may be defined as

$$
L_{\alpha}(t):=\int_{\mathbb{R}} \mathbf{1}_{[0, t]}(x) M(d x), \quad t \in[0,1] .
$$

Since $L_{\alpha}(t) \sim S_{\alpha}\left(t^{1 / \alpha}, 0,0\right)$, the logarithm of the characteristic function of $L_{\alpha}(t)$ is given by $\log \mathbb{E}_{\alpha}\left[e^{i \theta L_{\alpha}(t)}\right]=-t|\theta|^{\alpha}$.

A well-known representation of the stable Lévy process is its Ferguson-Klass-LePage series representation, based on the following sequences:

- $\left(\Gamma_{i}\right)_{i \geq 1}$ a sequence of arrival times of a Poisson process with unit arrival rate,

- $\left(V_{i}\right)_{i \geq 1}$ a sequence of i.i.d. random variables with uniform distribution on $[0,1]$, independent of $\left(\Gamma_{i}\right)_{i \geq 1}$, 
- $\left(\gamma_{i}\right)_{i \geq 1}$ a sequence of i.i.d. random variables with distribution $P\left(\gamma_{i}=1\right)=P\left(\gamma_{i}=-1\right)=$ $1 / 2$, independent of $\left(\Gamma_{i}\right)_{i \geq 1}$ and $\left(V_{i}\right)_{i \geq 1}$.

Consequently, the stable Lévy process $\left\{L_{\alpha}(t), t \in[0,1]\right\}$ admits the series representation:

$$
L_{\alpha}(t)=\sum_{i=1}^{\infty} \gamma_{i} C_{\alpha} \Gamma_{i}^{-1 / \alpha} \mathbf{1}_{[0, t]}\left(V_{i}\right)
$$

where $C_{\alpha}=\left(\int_{0}^{\infty} x^{-\alpha} \sin x d x\right)^{-1 / \alpha}$. For more details about Ferguson-Klass-LePage representations, we refer the reader to Ferguson and Klass (1972), Rosinski (1990) or Samorodnitsky and Taqqu (1994). The stable Lévy motion is therefore a càdlàg process, jumping at time $V_{i}$ with a jump of size $C_{\alpha} \Gamma_{i}^{-1 / \alpha}$, where the stability index $\alpha$ may be seen as a parameter regulating the size of the jumps.

The multistable processes are more flexible models because they allow us to consider a non constant index of stability $\alpha$. The size of the jumps will be governed by a function $\alpha(t)$ evolving with time. The first way to define such a process is to use the Ferguson-Klass-LePage representation of the stable processes, as in Le Guével and Lévy-Véhel (2012), replacing $\alpha$ by a function $\alpha:[0,1] \rightarrow(0,2)$. The first definition of the multistable Lévy motion is then

$$
L_{1}(t)=\sum_{i=1}^{\infty} \gamma_{i} C_{\alpha(t)} \Gamma_{i}^{-1 / \alpha(t)} \mathbf{1}_{[0, t]}\left(V_{i}\right)
$$

Since we have replaced $\alpha$ by $\alpha(t) \in(0,2)$, for each $t \in[0,1], Y(t)$ is a symmetric $\alpha(t)$-stable random variable $S_{\alpha(t)}\left(t^{1 / \alpha(t)}, 0,0\right)$ and $\log \mathbb{E}_{\alpha}\left[e^{i \theta L_{1}(t)}\right]=-t|\theta|^{\alpha(t)}$.

The second definition, developed by Falconer and Liu (2012), is inherited from the definition of multistable random measures $M_{\alpha(x)}$, where we have replaced again $\alpha$ by a function $\alpha(t)$. They defined the stochastic integral of $f$ with respect to a multistable random measure providing all its finite dimensional distributions. The Ferguson-Klass-LePage representation of the multistable Lévy motion resulting from this definition is

$$
L_{2}(t)=\sum_{i=1}^{\infty} \gamma_{i} C_{\alpha\left(V_{i}\right)} \Gamma_{i}^{-1 / \alpha\left(V_{i}\right)} \mathbf{1}_{[0, t]}\left(V_{i}\right)
$$

which satisfies $\log \mathbb{E}_{\alpha}\left[e^{i \theta L_{2}(t)}\right]=-\int_{0}^{t}|\theta|^{\alpha(x)} d x$. It follows that the two multistable processes have quite different properties. In particular, $L_{2}$ is a Markov process with independent increments, while $L_{1}$ has none of these two properties.

We already know that the two processes $L_{1}$ and $L_{2}$ are linked by the following formula (Le Guével, Lévy-Véhel, and Liu 2012, Theorem 8):

$$
L_{1}(t)=W(t)+L_{2}(t)
$$

where $W(t)=\int_{0}^{t} \sum_{i=1}^{+\infty} \gamma_{i} K_{i}(u) \mathbf{1}_{[0, u[}\left(V_{i}\right) d u$ and $K_{i}(u)=\frac{d\left(C_{\alpha(s)} \Gamma_{i}^{-1 / \alpha(s)}\right)}{d s}(u)$.

In this paper, we will consider the case of $L_{1}$ as well as the case of $L_{2}$. 


\section{Notations}

For the weak convergences, we will use the uniform metric, based on the Kolmogorov distance, denoted by $\kappa$. Recall that $\kappa$ is defined on the set of all probability measures on $\mathbb{R}$ by

$$
\kappa\left(\mu_{1}, \mu_{2}\right)=\sup _{x \in \mathbb{R}}\left|F_{\mu_{1}}(x)-F_{\mu_{2}}(x)\right|
$$

where $F_{\mu}$ is the cumulative distribution function with respect to $\mu$. From now on, we make the assumption that $\alpha$ is a $\mathcal{C}^{1}$ function which is ranging in $\left[\alpha_{*}, \alpha^{*}\right]$, a subset of $(1,2)$.

Given any $a$ and $b$ in $(0,1)$ and any $M>0$, we consider the subsets of continuously differentiable functions

$$
\Theta_{0}=\left\{\alpha:[a, b] \mapsto\left[\alpha_{*}, \alpha^{*}\right] \quad \mid \forall\left(t_{1}, t_{2}\right) \in[a, b]^{2}, \alpha\left(t_{1}\right)=\alpha\left(t_{2}\right)\right\}
$$

and

$$
\Theta_{1}=\left\{\alpha:[a, b] \mapsto\left[\alpha_{*}, \alpha^{*}\right] \quad \mid \exists\left(t_{1}, t_{2}\right) \in[a, b]^{2}, \alpha\left(t_{1}\right) \neq \alpha\left(t_{2}\right) \text { and }\left\|\alpha^{\prime}\right\|_{\infty} \leq M\right\} .
$$

If $\alpha$ is not continuously differentiable, some results will no longer be valid, because we can not apply the mean value theorem anymore. The proofs may be adapted if $\alpha$ is Hölder continuous instead of Lipschitz. On the other side, the interesting case of change point detection problem with a piecewise constant function $\alpha$ is rather more challenging. This would be an interesting further development that would require new methods.

For simplicity of notation, we will write $\alpha$ instead of $\alpha(t)$ if $\alpha \in \Theta_{0}$. If $Z$ is a standard symmetric $\alpha$-stable random variable $\left(Z \sim S_{\alpha}(1,0,0)\right)$, we know that if $\alpha \in(1,2)$,

$$
\mu(\alpha):=\mathbb{E}_{\alpha}[\log |Z|]=\Gamma^{\prime}(1)\left(1-\frac{1}{\alpha}\right)
$$

and

$$
\operatorname{var}_{\alpha}(\log |Z|)=\frac{\pi^{2}}{6 \alpha^{2}}+\frac{\pi^{2}}{12}
$$

Therefore we introduce the two functions $v(x)=\frac{\pi^{2}}{6 x^{2}}+\frac{\pi^{2}}{12}$ and $\sigma^{2}(x)=(v(x) \vee v(2)) \wedge v(1)$, and we will denote

$$
h(t):=\frac{1}{\alpha(t)}
$$

We define $X_{k, N}$ to be the increment $X\left(\frac{k+1}{N}\right)-X\left(\frac{k}{N}\right)$ of a process $X, N \in \mathbb{N} \backslash\{0\}, k=1, \ldots, N$ and we denote $t_{k}=\frac{k}{N}$. We also introduce the process

$$
U_{k, N}(t)=\sum_{i=1}^{\infty} \gamma_{i} C_{\alpha\left(t_{k}\right)} \Gamma_{i}^{-1 / \alpha\left(t_{k}\right)} \mathbf{1}_{[0, t]}\left(V_{i}\right)
$$

and its increments

$$
U_{k, N}:=U_{k, N}\left(t_{k+1}\right)-U_{k, N}\left(t_{k}\right)=\sum_{i=1}^{\infty} \gamma_{i} C_{\alpha\left(t_{k}\right)} \Gamma_{i}^{-1 / \alpha\left(t_{k}\right)} \mathbf{1}_{\left(t_{k}, t_{k+1}\right]}\left(V_{i}\right)
$$


Note that for $k \neq j$, since $\left(t_{k}, t_{k+1}\right] \cap\left(t_{j}, t_{j+1}\right]=\varnothing, U_{k, N}$ is independent of $U_{j, N}$ (see Le Guével and Lévy-Véhel (2012, Proposition 6.1) for the expression of the characteristic function of $\left.\left(U_{k, N}, U_{j, N}\right)\right)$. Since $U_{k, N}(t)$ is a standard symmetric $\alpha\left(t_{k}\right)$-stable Lévy process, we know that $N^{1 / \alpha\left(t_{k}\right)} U_{k, N} \sim S_{\alpha\left(t_{k}\right)}(1,0,0)$ and

$$
\operatorname{var}_{\alpha}\left(\log \left|U_{k, N}\right|\right)=\operatorname{var}_{\alpha}\left(\log \left|N^{1 / \alpha\left(t_{k}\right)} U_{k, N}\right|\right)=\sigma^{2}\left(\alpha\left(t_{k}\right)\right)
$$

In the sequel, for estimation and testing problems, $(n(N))_{N \in \mathbb{N}}$ will be a sequence taking even integer values such that $n(N) \leq N$. Several rates of convergence will depend on the sequence $\xi_{N}:=\sqrt{n(N)}\left(\Gamma^{\prime}(1)+\log N\right)$. For $t \in[a, b] \subset(0,1)$, with the observations $\left(X_{k, N}\right)_{k}$, we introduce an estimator of $h(t)$ by

$$
\hat{h}_{N}(t)=\frac{1}{n(N)} \sum_{k=\lfloor N t\rfloor-\frac{n(N)}{2}}^{\lfloor N t\rfloor+\frac{n(N)}{2}-1} \frac{\Gamma^{\prime}(1)-\log \left|X_{k, N}\right|}{\Gamma^{\prime}(1)+\log N}
$$

where $\lfloor$.$\rfloor is the floor function (see Le Guével (2013) for the properties of this estimator). We$ will also consider a slight modification using the process $U$ (which will not be observed) with

$$
\hat{h}_{N}^{U}(t)=\frac{1}{n(N)} \sum_{k=\lfloor N t\rfloor-\frac{n(N)}{2}}^{\lfloor N t\rfloor+\frac{n(N)}{2}-1} \frac{\Gamma^{\prime}(1)-\log \left|U_{k, N}\right|}{\Gamma^{\prime}(1)+\log N}
$$

and we shall write $\bar{h}(t)$ for its expectation $\mathbb{E}_{\alpha}\left[\hat{h}_{N}^{U}(t)\right]$, that is

$$
\bar{h}(t)=\frac{1}{n(N)} \sum_{k=\lfloor N t\rfloor-\frac{n(N)}{2}}^{\lfloor N t\rfloor+\frac{n(N)}{2}-1} h\left(\frac{k}{N}\right) .
$$

We also introduce a plug-in estimator of $\sigma^{2}(\alpha(t))$ with

$$
\hat{\sigma}^{2}(t)=\sigma^{2}\left(\frac{1}{\hat{h}_{N}(t)}\right) .
$$

Our test is based on straightforward properties, listed below, of the estimator of $h$ which are satisfied when the observed process is a standard stable Lévy motion, that is when $\alpha$ is no longer varying $\left(\alpha \in \Theta_{0}\right)$ :

- If $\alpha \in \Theta_{0}$, for all $N \in \mathbb{N}^{*}$ and $t_{0} \in[a, b]$,

$$
\mathbb{E}_{\alpha}\left[\hat{h}_{N}\left(t_{0}\right)\right]=h=\frac{1}{\alpha} \text { and } \operatorname{var}_{\alpha}\left[\hat{h}_{N}\left(t_{0}\right)\right]=\frac{\sigma^{2}(\alpha)}{n(N)\left(\Gamma^{\prime}(1)+\log N\right)^{2}} .
$$

Furthermore, if $\lim _{N \rightarrow+\infty} n(N)=+\infty$ and $\lim _{N \rightarrow+\infty} \frac{n(N)}{N}=0$,

$$
\lim _{N \rightarrow+\infty} \frac{\left(\Gamma^{\prime}(1)+\log N\right) \sqrt{n(N)}}{\sqrt{\sigma^{2}(\alpha)}}\left(\hat{h}_{N}\left(t_{0}\right)-h\right) \stackrel{d}{=} \mathcal{N}(0,1) .
$$


- There exists $c_{0}>0$ such that for all $\varepsilon>0$ and $t_{0} \in[a, b]$,

$$
\sup _{\alpha \in \Theta_{0}} \mathbb{P}_{\alpha}\left(\left|\hat{h}_{N}\left(t_{0}\right)-h\right|>2 \varepsilon\right) \leq \frac{c_{0}^{n(N)}}{e^{\varepsilon n(N)\left(\Gamma^{\prime}(1)+\log N\right)}},
$$

whereby for every $t_{0} \in[a, b]$ and $\alpha \in \Theta_{0}$, if $\lim _{N \rightarrow+\infty} n(N)=+\infty$,

$$
\lim _{N \rightarrow+\infty} \hat{h}_{N}\left(t_{0}\right) \stackrel{\mathbb{P}_{\alpha} \text { a.s. }}{=} h=\frac{1}{\alpha} .
$$

Finally, in all the paper, $K$ stands for an absolute positive constant, and $K^{*}$ will be a positive constant which depends on $\Theta_{0}$ or $\Theta_{1}$, that is $a, b, \alpha_{*}, \alpha^{*}$ and $M$.

\section{Testing the multistability}

We assume that the observation of $X=L_{1}$ or $X=L_{2}$ consists of one realization of the points $X\left(\frac{k}{N}\right), N \in \mathbb{N}^{*}, k=1, \ldots, N$. Our purpose is to solve the following testing problem

$$
\left(H_{0}\right): \alpha \in \Theta_{0} \quad \text { vs }\left(H_{1}\right): \alpha \in \Theta_{1} .
$$

Set $\left(t_{0}, t_{1}\right) \in(a, b)^{2}, t_{0} \neq t_{1}$, and assume that $\left(t_{0}-\frac{n(N)}{2 N}, t_{0}+\frac{n(N)}{2 N}\right) \cap\left(t_{1}-\frac{n(N)}{2 N}, t_{1}+\frac{n(N)}{2 N}\right)=\varnothing$ (this is always available for $N$ large enough as soon as $\lim _{N \rightarrow+\infty} \frac{n(N)}{N}=0$ ). Our test is based on the statistic

$$
T_{N}=\frac{n(N)\left(\Gamma^{\prime}(1)+\log N\right)^{2}}{\hat{\sigma}^{2}\left(t_{1}\right)(b-a)} \int_{a}^{b}\left|\hat{h}_{N}(t)-\hat{h}_{N}\left(t_{0}\right)\right|^{2} d t .
$$

According to the asymptotic distribution of $T_{N}$ under the null hypothesis, we obtain an asymptotic consistent test with asymptotic size $\beta \in(0,1)$ choosing the rejection region

$$
\mathrm{R}_{\mathrm{c}}:=\left[q_{\beta},+\infty\right)
$$

where $q_{\beta}$ is the $1-\beta$ quantile of the distribution $1+\chi^{2}(1)$, and $\chi^{2}(1)$ is the $\chi^{2}$ distribution with one degree of freedom.

The following statements are our main results. The first theorem exhibits the asymptotic distribution of the statistic $T_{N}$ under the null hypothesis, providing a uniform bound for the size of the test.

Theorem 1 (Null hypothesis). Let $P_{N}^{\alpha}$ be the distribution of $T_{N}$ and $P_{1+\chi^{2}}$ be the distribution of $1+\chi^{2}(1)$, where $\chi^{2}(1)$ stands for the $\chi^{2}$ distribution with one degree of freedom. There exists a constant $K^{*}>0$ such that for all $N \geq 1$,

$$
\sup _{\alpha \in \Theta_{0}} \kappa\left(P_{N}^{\alpha}, P_{1+\chi^{2}}\right) \leq K^{*}\left(\frac{1}{\xi_{N}}+\frac{1}{\sqrt{n(N)}}+\left(\sqrt{\frac{n(N)}{N}}+\frac{1}{\xi_{N}}+\frac{1}{\sqrt{\lfloor N b\rfloor-\lfloor N a\rfloor-n(N)}}\right)^{1 / 3}\right) .
$$


Thereby there exists a constant $K^{*}>0$ such that for every fixed level $\beta \in(0,1)$ in the testing problem and every $N \geq 1$,

$\sup _{\alpha \in \Theta_{0}} \mathbb{P}_{\alpha}\left(T_{N} \in \mathrm{R}_{\mathrm{c}}\right) \leq \beta+K^{*}\left(\frac{1}{\xi_{N}}+\frac{1}{\sqrt{n(N)}}+\left(\sqrt{\frac{n(N)}{N}}+\frac{1}{\xi_{N}}+\frac{1}{\sqrt{\lfloor N b\rfloor-\lfloor N a\rfloor-n(N)}}\right)^{1 / 3}\right)$.

Furthermore, if $\lim _{N \rightarrow+\infty} n(N)=+\infty$ and $\lim _{N \rightarrow+\infty} \frac{n(N)}{N}=0$, then the following convergence in distribution occurs for all $\alpha \in \Theta_{0}$ :

$$
\lim _{N \rightarrow+\infty} T_{N} \stackrel{d}{=} 1+\chi^{2}(1)
$$

The next theorem deals with the convergence of the test statistic $T_{N}$ under the alternative hypothesis. We obtain a consistent procedure under mild conditions on $n(N)$.

Theorem 2 (Alternative hypothesis for $L_{1}$ ). Assume that the observed process is $L_{1}$ (i.e. $\left.X=L_{1}\right)$. Define

$$
\chi_{N}:=\frac{1}{\xi_{N}}\left(T_{N}-\frac{\xi_{N}^{2}}{\hat{\sigma}^{2}\left(t_{1}\right)} \frac{1}{b-a} \int_{a}^{b}\left|h(t)-h\left(t_{0}\right)\right|^{2} d t\right)
$$

and consider $\chi$, a random variable normally distributed $\mathcal{N}\left(0, \sigma_{\chi}^{2}\right)$ with

$$
\sigma_{\chi}^{2}=\frac{4 \sigma^{2}\left(\alpha\left(t_{0}\right)\right)}{\sigma^{4}\left(\alpha\left(t_{1}\right)\right)(b-a)^{2}}\left(\int_{a}^{b}\left(h(t)-h\left(t_{0}\right)\right) d t\right)^{2} .
$$

Let $P_{\chi_{N}}$ be the distribution of $\chi_{N}, P_{\chi}$ be the distribution of $\chi$, and let $\eta \in\left(0, \frac{1}{2}\right)$. There exists a positive constant $K_{\eta}^{*}$ such that for all $\alpha \in \Theta_{1}$ and $N \geq 1$,

$$
\kappa\left(P_{\chi_{N}}, P_{\chi}\right) \leq K_{\eta}^{*}\left(\frac{1}{\sqrt{n(N)}}+\frac{1}{\sqrt{\sigma_{\chi}}} \sqrt{\frac{\xi_{N}}{N^{\left(1-\frac{1}{\alpha_{*}}\right) \frac{\eta}{2}}}+\xi_{N} \frac{n(N)}{N}+\left(\frac{n(N)}{N}\right)^{\frac{1}{2}}+\frac{1}{\xi_{N}}}\right)
$$

Thereby if $n(N)=N^{\gamma}$ with $\gamma \in\left(0, \frac{\alpha_{*}-1}{4 \alpha_{*}}\right)$, then for any $\alpha \in \Theta_{1}$,

$$
\lim _{N \rightarrow+\infty} \chi_{N} \stackrel{d}{=} \chi
$$

and for a fixed level $\beta \in(0,1)$ in the testing problem, there exists a constant $K_{\gamma}^{*}>0$ such that for all $\alpha \in \Theta_{1}$ and $N \geq 1$,

$\mathbb{P}_{\alpha}\left(T_{N} \in \mathrm{R}_{c}\right) \geq \mathbb{P}_{\alpha}\left(\chi \leq \frac{\xi_{N}}{3(b-a)} \int_{a}^{b}\left|h(t)-h\left(t_{0}\right)\right|^{2} d t-\frac{q_{\beta}}{\xi_{N}}\right)-K_{\gamma}^{*} \frac{\sqrt{\log N}}{N^{\gamma / 4}}\left(\frac{1}{b-a}\left|\int_{a}^{b}\left(h(t)-h\left(t_{0}\right)\right) d t\right|\right)^{-\frac{1}{2}}$ 
If $h\left(t_{0}\right)=\frac{1}{b-a} \int_{a}^{b} h(t) d t, \lim _{N \rightarrow+\infty} \chi_{N} \stackrel{\mathbb{P}_{\alpha}}{=} 0$ where the convergence is in probability. In fact it appears in the proof of Theorem 2 that in that case the following convergence occurs

$$
\lim _{N \rightarrow+\infty} T_{N}-\frac{\xi_{N}^{2}}{\hat{\sigma}^{2}\left(t_{1}\right)} \frac{1}{b-a} \int_{a}^{b}\left|h(t)-h\left(t_{0}\right)\right|^{2} d t \stackrel{d}{=} \frac{1}{b-a} \int_{a}^{b} \frac{\sigma^{2}(\alpha(t))}{\sigma^{2}\left(\alpha\left(t_{1}\right)\right)} d t+\frac{\sigma^{2}\left(\alpha\left(t_{0}\right)\right)}{\sigma^{2}\left(\alpha\left(t_{1}\right)\right)} \chi^{2}(1),
$$

and we may obtain a similar bound for the Kolmogorov's distance between the two distributions.

The last result deals with the multistable Lévy process with independent increments $L_{2}$ under the alternative hypothesis.

Theorem 3 (Alternative hypothesis for $L_{2}$ ). Assume that the observed process is $L_{2}$ (i.e. $\left.X=L_{2}\right)$, that $\left(\frac{n(N) \log N}{N}\right)_{N}$ is bounded and $n(N) \geq 256$. Then there exists a constant $K^{*}>0$ such that for all $\alpha \in \Theta_{1}$ and $x>0$,

$$
\mathbb{P}_{\alpha}\left(T_{N} \leq x\right) \leq e^{K^{*} \sqrt{x}}\left(K^{*}\right) \sqrt{n(N)} e^{-\frac{\xi_{N}}{b-a} \int_{a}^{b}\left|h(t)-h\left(t_{0}\right)\right| d t}
$$

Thereby for a fixed level $\beta \in(0,1)$ in the testing problem, there exists a constant $K_{\beta}>0$ such that for every $\alpha \in \Theta_{1}$,

$$
\mathbb{P}_{\alpha}\left(T_{N} \in \mathrm{R}_{\mathrm{c}}\right) \geq 1-K_{\beta}^{\sqrt{n(N)}} e^{-\frac{\xi_{N}}{b-a} \int_{a}^{b}\left|h(t)-h\left(t_{0}\right)\right| d t}
$$

Comparing with Theorem 2, we see that the power is asymptotically greater if $X=L_{2}$ because we can take advantage of less correlated data in that case.

\section{Technical lemmas}

We set in this section a list of lemmas used in the proofs of the main theorems. The first one is a combinatory result, given without the proof.

Lemma 4. For every sequence $\left(W_{k, j}\right)_{(k, j) \in \mathbb{Z}^{2}}$,

$$
\begin{aligned}
\sum_{j=\lfloor N a\rfloor+1}^{\lfloor N b\rfloor-1} \sum_{k=j-\frac{n(N)}{2}}^{j+\frac{n(N)}{2}} W_{k, j} & =\sum_{k=\lfloor N a\rfloor+1}^{\lfloor N a\rfloor+\frac{n(N)}{2}-1} \sum_{j=\lfloor N a\rfloor+1}^{k+\frac{n(N)}{2}} W_{k, j}+\sum_{k=\lfloor N a\rfloor+\frac{n(N)}{2}}^{\lfloor N b\rfloor-\frac{n(N)}{2}-1} \sum_{j=k-\frac{n(N)}{2}+1}^{k+\frac{n(N)}{2}} W_{k, j} \\
& +\sum_{k=\lfloor N b\rfloor-\frac{n(N)}{2}}^{\lfloor N b\rfloor+\frac{n(N)}{2}-2} \sum_{j=k-\frac{n(N)}{2}+1}^{\lfloor N b\rfloor-1} W_{k, j} .
\end{aligned}
$$

The next lemma provides a uniform upper bound of the moment of the logarithm of standard $\alpha$-stable random variables. 
Lemma 5. Let $\eta>0$ and $\left[\alpha_{*}, \alpha^{*}\right] \subset(1,2)$.

$$
\sup _{\alpha \in\left[\alpha_{*}, \alpha^{*}\right]} \mathbb{E}_{\alpha}\left[|\log | W \mid-\mathbb{E}_{\alpha}\left[\left.\log [|W|]\right|^{\eta}\right]<+\infty\right.
$$

where $W$ is a standard symmetric $\alpha$-stable random variable.

In the next lemma, we provide a uniform upper bound of the moments of the inverse of the increments for the three processes $L_{1}, L_{2}$ and $U$.

Lemma 6. For all $\eta \in\left(0, \frac{1}{2}\right)$,

$$
\sup _{N} \sup _{k=1, \ldots, N} \sup _{\alpha \in \Theta_{1}} N^{-2 h\left(t_{k}\right) \eta} \mathbb{E}_{\alpha}\left[\left|X_{k, N}\right|^{-2 \eta}\right]<+\infty
$$

where $X=L_{1}, X=L_{2}$ or $X=U$, and $h(t)=\frac{1}{\alpha(t)}$.

We set now a control of the variances of $\hat{h}_{N}^{U}(t)$ and $\hat{h}_{N}(t)$.

Lemma 7. Assume that $X=L_{1}$. For all $\eta \in\left(0, \frac{1}{2}\right)$,

$$
\sup _{N} \sup _{\alpha \in \Theta_{1}} \sup _{t \in[a, b]} \mathbb{E}_{\alpha}\left[\xi_{N}^{2}\left|\bar{h}(t)-\hat{h}_{N}^{U}(t)\right|^{2}\right]<+\infty
$$

and

$$
\sup _{N} \sup _{\alpha \in \Theta_{1}} \sup _{t \in[a, b]} \mathbb{E}_{\alpha}\left[N^{\left(1-\frac{1}{\alpha_{*}}\right) \eta}\left|\hat{h}_{N}(t)-\hat{h}_{N}^{U}(t)\right|^{2}\right]<+\infty .
$$

The last lemma states that the estimator of the function $h$ has exponential moments if the observed multistable process is $L_{2}$.

Lemma 8. Assume that $X=L_{2}$ and $\left(\frac{n(N) \log N}{N}\right)_{N}$ is bounded. Then

$$
\sup _{N} \sup _{\alpha \in \Theta_{1}} \sup _{t \in[a, b]} \frac{\lfloor N t\rfloor+\frac{n(N)}{\sup ^{2}}-1}{k=\lfloor N t\rfloor-\frac{n(N)}{2}} \mathbb{E}_{\alpha}\left[N^{h(t)}\left|X_{k, N}\right|\right]<+\infty
$$

and if $n(N) \geq 256$,

$$
\sup _{N} \sup _{\alpha \in \Theta_{1}} \sup _{t \in[a, b]} \frac{\log \mathbb{E}_{\alpha}\left[e^{2\left|W_{N}(t)\right|}\right]}{\sqrt{n(N)}}<+\infty
$$

where $W_{N}(t)=\xi_{N}\left(\hat{h}_{N}(t)-h(t)\right)$.

\section{Proofs}

Proof of Lemma 5 Let $\alpha \in\left[\alpha_{*}, \alpha^{*}\right], W$ be a standard $\alpha$-stable random variable, and put $\mu(\alpha)=\mathbb{E}_{\alpha}[\log |W|]=\Gamma^{\prime}(1)\left(1-\frac{1}{\alpha}\right)$. 


$$
\begin{aligned}
\mathbb{E}_{\alpha}\left[|\log | W \mid-\mathbb{E}_{\alpha}\left[\left.\log [|W|]\right|^{\eta}\right]\right. & =\int_{0}^{+\infty} \mathbb{P}_{\alpha}\left(|\log | W|-\mu(\alpha)|>x^{1 / \eta}\right) d x \\
& \leq 1+\int_{1}^{+\infty} \mathbb{P}_{\alpha}\left(|W| \geq e^{x^{1 / \eta}} e^{\mu(\alpha)}\right) d x+\int_{1}^{+\infty} \mathbb{P}_{\alpha}\left(|W| \leq e^{-x^{1 / \eta}} e^{\mu(\alpha)}\right) d x .
\end{aligned}
$$

Since the characteristic function of $W$ is $\mathbb{E}_{\alpha}\left[e^{i y W}\right]=e^{-|y|^{\alpha}}$, Parseval's inversion formula provides an upper bound for the last term of the right handside of the previous inequality. Moreover, the Markov inequality for the first term leads to

$$
\begin{aligned}
\mathbb{E}_{\alpha}\left[|\log | W|-\mu(\alpha)|^{\eta}\right] & \leq 1+e^{-\mu(\alpha)} \mathbb{E}_{\alpha}[|W|] \int_{1}^{+\infty} e^{-x^{1 / \eta}} d x+\frac{1}{\pi} \int_{1}^{+\infty} \int_{\mathbb{R}} e^{-|y|^{\alpha}} \frac{\sin \left(e^{-x^{1 / \eta}} e^{\mu(\alpha)} y\right)}{y} d y d x \\
& \leq 1+e^{-\mu(\alpha)} \mathbb{E}_{\alpha}[|W|] \int_{1}^{+\infty} e^{-x^{1 / \eta}} d x+\frac{e^{\mu(\alpha)}}{\pi} \int_{1}^{+\infty} \int_{\mathbb{R}}\left(e^{-|y|}+e^{-|y|^{2}}\right) e^{-x^{1 / \eta}} d y d x .
\end{aligned}
$$

We obtain (23) since $\mathbb{E}_{\alpha}[|W|]=\frac{2}{\pi} \Gamma\left(1-\frac{1}{\alpha}\right)$ (see Samorodnitsky and Taqqu 1994, property 1.2.17) and $\alpha \in\left[\alpha_{*}, \alpha^{*}\right]$.

Proof of Lemma 6 We begin the proof showing that for $X=L_{1}, X=L_{2}$ or $X=U$,

$$
\sup _{N} \sup _{k=1, \ldots, N} \int_{\mathbb{R}} \phi_{N}^{X}\left(N^{h\left(t_{k}\right)} \theta\right) d \theta<\infty
$$

where $\phi_{N}^{X}$ is the characteristic function of $X_{k, N}$. Recall that $t_{k}=\frac{k}{N}$ and consider first the case $X=L_{2}$. We know from Falconer and Liu (2012) that the characteristic function of $X_{k, N}$ is given by $\phi_{N}^{X}(\theta)=e^{-\int_{t_{k}}^{t_{k+1}}|\theta|^{\alpha(x)} d x}$. Then we use the fact that $\alpha \geq 1$ to obtain

$$
\begin{aligned}
\int_{\mathbb{R}} \phi_{N}^{X}\left(N^{h\left(t_{k}\right)} \theta\right) d \theta & =\int_{\mathbb{R}} e^{-\int_{t_{k}}^{t_{k+1}}\left|N^{h\left(t_{k}\right)} \theta\right|^{\alpha(x)} d x} d \theta \\
& \leq 2+2 \int_{1}^{+\infty} e^{-\theta \int_{t_{k}}^{t_{k+1}} N^{h\left(t_{k}\right) \alpha(x)} d x} d \theta \\
& \leq 2+\frac{2}{\int_{t_{k}}^{t_{k+1}} N^{h\left(t_{k}\right) \alpha(x)} d x} \\
& =2+\frac{2}{N \int_{t_{k}}^{t_{k+1}} N^{h\left(t_{k}\right) \alpha(x)-1} d x} .
\end{aligned}
$$

If $\alpha \in \Theta_{1}$, the mean value theorem yields $\left|h\left(t_{k}\right) \alpha(x)-1\right|=\left|\frac{\alpha(x)-\alpha\left(t_{k}\right)}{\alpha\left(t_{k}\right)}\right| \leq \frac{\left\|\alpha^{\prime}\right\|_{\infty}}{N \alpha_{*}} \leq \frac{M}{N \alpha_{*}}$ so $N^{h\left(t_{k}\right) \alpha(x)-1} \geq N^{-M /\left(N \alpha_{*}\right)}$ and

$$
\int_{\mathbb{R}} \phi_{N}^{X}\left(N^{h\left(t_{k}\right)} \theta\right) d \theta \leq 1+2 \sup _{N} N^{M /\left(N \alpha_{*}\right)},
$$


hence (29). We consider now the case $X=L_{1}$. For $t \in[a, b]$, recall that $C_{\alpha(t)}=\left(\int_{0}^{\infty} x^{-\alpha(t)} \sin x d x\right)^{-1 / \alpha(t)}$ and write

$$
A_{k}=\frac{C_{\alpha\left(t_{k+1}\right)}}{2 y^{1 / \alpha\left(t_{k+1}\right)}} \mathbf{1}_{\left[t_{k}, t_{k+1}\right]}(x)
$$

and

$$
B_{k}=\left(\frac{C_{\alpha\left(t_{k+1}\right)}}{2 y^{1 / \alpha\left(t_{k+1}\right)}}-\frac{C_{\alpha\left(t_{k}\right)}}{2 y^{1 / \alpha\left(t_{k}\right)}}\right) \mathbf{1}_{\left[0, t_{k}\right]}(x) .
$$

We know from Le Guével and Lévy-Véhel (2012) that the characteristic function of $X_{k, N}=$ $L_{1}\left(t_{k+1}\right)-L_{1}\left(t_{k}\right)$ is given by

$$
E\left[e^{i \theta X_{k, N}}\right]=e^{-2 \int_{0}^{1} \int_{0}^{+\infty} \sin ^{2}\left(\theta\left(A_{k}+B_{k}\right)\right) d x d y} .
$$

It follows from the equality $\int_{0}^{\infty} \frac{\sin ^{2}(z)}{z^{1+\alpha}} d z=\frac{2^{\alpha-1}}{\alpha} C_{\alpha}^{-\alpha}$ that

$$
\begin{aligned}
\int_{\mathbb{R}} \phi_{N}^{X}\left(N^{h\left(t_{k}\right)} \theta\right) d \theta & \leq 2+2 \int_{1}^{+\infty} e^{-2} \int_{t_{k}}^{t_{k+1}+\infty} \int_{0}^{+\infty} \sin ^{2}\left(N^{h\left(t_{k}\right)} \theta A_{k}\right) d x d y \\
& =2+2 \int_{1}^{+\infty} e^{-\theta^{\alpha\left(t_{k+1}\right)}} d \theta \\
& \leq 2+2 \int_{1}^{+\infty}\left(e^{-\theta}+e^{-\theta^{2}}\right) d \theta
\end{aligned}
$$

which implies (29). Finally, the characteristic function of $U_{k, N}$ is $\phi_{N}^{U}(\theta)=e^{-\frac{|\theta| \alpha\left(t_{k}\right)}{N}}$ so we derive (29) since $\alpha \in\left[\alpha_{*}, \alpha^{*}\right]$.

Now we consider that $X$ is either $L_{1}, L_{2}$ or $U$. Parseval's inversion formula yields

$$
\begin{aligned}
\mathbb{P}_{\alpha}\left(\left|X_{k, N}\right| \leq y\right) & =\frac{1}{\pi} \int_{\mathbb{R}} \frac{\sin (\xi y)}{\xi} \phi_{N}^{X}(\xi) d \xi \\
& =\frac{1}{\pi} \int_{\mathbb{R}} \frac{\sin \left(N^{h\left(t_{k}\right)} \theta y\right)}{\theta} \phi_{N}^{X}\left(N^{h\left(t_{k}\right)} \theta\right) d \theta \\
& \leq \frac{N^{h\left(t_{k}\right)}}{\pi} y \int_{\mathbb{R}} \phi_{N}^{X}\left(N^{h\left(t_{k}\right)} \theta\right) d \theta \\
& \leq K N^{h\left(t_{k}\right)} y \sup _{N} \sup _{k=1, \ldots, N} \int_{\mathbb{R}} \phi_{N}^{X}\left(N^{h\left(t_{k}\right)} \theta\right) d \theta .
\end{aligned}
$$


We obtain to conclude for $\eta \in\left(0, \frac{1}{2}\right)$

$$
\begin{aligned}
\mathbb{E}_{\alpha}\left[\left|X_{k, N}\right|^{-2 \eta}\right] & =\int_{0}^{\infty} \mathbb{P}_{\alpha}\left(\left|X_{k, N}\right| \leq N^{-h\left(t_{k}\right)} v^{-\frac{1}{2 \eta}}\right) N^{2 h\left(t_{k}\right) \eta} d v \\
& \leq N^{2 h\left(t_{k}\right) \eta}\left(1+\int_{1}^{\infty} \mathbb{P}_{\alpha}\left(\left|X_{k, N}\right| \leq N^{-h\left(t_{k}\right)} v^{-\frac{1}{2 \eta}}\right) d v\right)
\end{aligned}
$$

and

$$
\sup _{\alpha \in \Theta_{1}} N^{-2 h\left(t_{k}\right) \eta} \mathbb{E}_{\alpha}\left[\left|X_{k, N}\right|^{-2 \eta}\right] \leq 1+K \sup _{N} \sup _{k=1, \ldots, N} \int_{\mathbb{R}} \phi_{N}^{X}\left(N^{h\left(t_{k}\right)} \theta\right) d \theta \int_{1}^{\infty} v^{-\frac{1}{2 \eta}} d v
$$

which is $(24)$.

Proof of Lemma 7 Let $\alpha \in \Theta_{1}$ and $t \in[a, b]$. From the definition $(9)$ of $\hat{h}_{N}^{U}(t)$, we have

$$
\bar{h}(t)-\hat{h}_{N}^{U}(t)=\frac{1}{n(N)} \sum_{k=\lfloor N t\rfloor-\frac{n(N)}{2}}^{\lfloor N t\rfloor+\frac{n(N)}{2}-1} \frac{\log \left|U_{k, N}\right|-\mathbb{E}_{\alpha}\left[\log \left|U_{k, N}\right|\right]}{\Gamma^{\prime}(1)+\log N} .
$$

We use the independence of the random variables $\left(U_{k, N}\right)_{k}$ to obtain

$$
\operatorname{var}_{\alpha}\left(\hat{h}_{N}^{U}(t)\right)=\frac{1}{\xi_{N}^{2}} \sum_{k=\lfloor N t\rfloor-\frac{n(N)}{2}}^{\lfloor N t\rfloor+\frac{n(N)}{2}-1} v\left(\alpha\left(t_{k}\right)\right) \leq \frac{v(1)}{\xi_{N}^{2}}
$$

that is (25). To prove (26), write for $\alpha \in \Theta_{1}$ and $t \in[a, b]$,

$$
\hat{h}_{N}(t)-\hat{h}_{N}^{U}(t)=\frac{1}{n(N)} \sum_{k=\lfloor N t\rfloor-\frac{n(N)}{2}}^{\lfloor N t\rfloor+\frac{n(N)}{2}-1} \log \left|\frac{U_{k, N}}{X_{k, N}}\right|
$$

whereby

$$
\mathbb{E}_{\alpha}\left[\left|\hat{h}_{N}(t)-\hat{h}_{N}^{U}(t)\right|^{2}\right]^{\frac{1}{2}} \leq \frac{1}{n(N)} \sum_{k=\lfloor N t\rfloor-\frac{n(N)}{2}}^{\lfloor N t\rfloor+\frac{n(N)}{2}-1} \mathbb{E}_{\alpha}\left[\left.|\log | \frac{U_{k, N}}{X_{k, N}}\right|^{2}\right]^{\frac{1}{2}}
$$

Let $\eta \in\left(0, \frac{1}{2}\right)$ and fix $K_{\eta}>0$ such that for all $x \in \mathbb{R},(\log x)^{2} \mathbf{1}_{x \geq 1} \leq K_{\eta}|x-1|^{\eta}$. We deduce from the inequality

$$
\begin{aligned}
|\log | x||^{2} & =|\log x|^{2} \mathbf{1}_{x \geq 1}+\left|\log \frac{1}{x}\right|^{2} \mathbf{1}_{\frac{1}{x} \geq 1} \\
& \leq K_{\eta}\left(|x-1|^{\eta}+\left|\frac{1}{x}-1\right|^{\eta}\right)
\end{aligned}
$$

that $\left.|\log | \frac{U_{k, N}}{X_{k, N}}\right|^{2} \leq K_{\eta}\left|U_{k, N}-X_{k, N}\right|^{\eta}\left(\left|\frac{1}{X_{k, N}}\right|^{\eta}+\left|\frac{1}{U_{k, N}}\right|^{\eta}\right)$ which implies 


$$
\begin{aligned}
\mathbb{E}_{\alpha}\left[|\log | \frac{U_{k, N}}{X_{k, N}}||^{2}\right] & \leq K_{\eta} \mathbb{E}_{\alpha}\left[\left|U_{k, N}-X_{k, N}\right|^{2 \eta}\right]^{\frac{1}{2}} \mathbb{E}_{\alpha}\left[\left(\left|X_{k, N}\right|^{-\eta}+\left|U_{k, N}\right|^{-\eta}\right)^{2}\right]^{\frac{1}{2}} \\
& \leq K_{\eta} \mathbb{E}_{\alpha}\left[\left|U_{k, N}-X_{k, N}\right|\right]^{\eta}\left(\mathbb{E}_{\alpha}\left[\left|X_{k, N}\right|^{-2 \eta}\right]^{\frac{1}{2}}+\mathbb{E}_{\alpha}\left[\left|U_{k, N}\right|^{-2 \eta}\right]^{\frac{1}{2}}\right) \\
& \leq K_{\eta}^{*} N^{h\left(t_{k}\right) \eta} \mathbb{E}_{\alpha}\left[\left|U_{k, N}-X_{k, N}\right|\right]^{\eta},
\end{aligned}
$$

where we have used (24) for the last inequality. Let us control $\mathbb{E}_{\alpha}\left[\left|U_{k, N}-X_{k, N}\right|\right]$. To this end, thanks to the definition of $L_{1}$ and (6), we get

$$
X_{k, N}-U_{k, N}=\sum_{i=1}^{\infty} \gamma_{i}\left(C_{\alpha\left(t_{k+1}\right)} \Gamma_{i}^{-1 / \alpha\left(t_{k+1}\right)}-C_{\alpha\left(t_{k}\right)} \Gamma_{i}^{-1 / \alpha\left(t_{k}\right)}\right) \mathbf{1}_{\left[0, t_{k+1}\right]}\left(V_{i}\right) .
$$

The mean value theorem yields that there exists a random variables $c_{i} \in\left(\alpha\left(t_{k}\right), \alpha\left(t_{k+1}\right)\right)$ (or $\left.c_{i} \in\left(\alpha\left(t_{k+1}\right), \alpha\left(t_{k}\right)\right)\right)$ such that

$$
C_{\alpha\left(t_{k+1}\right)} \Gamma_{i}^{-1 / \alpha\left(t_{k+1}\right)}-C_{\alpha\left(t_{k}\right)} \Gamma_{i}^{-1 / \alpha\left(t_{k}\right)}=\left(\alpha\left(t_{k+1}\right)-\alpha\left(t_{k}\right)\right) \frac{d}{d u}\left(C_{u} \Gamma_{i}^{-1 / u}\right)\left(c_{i}\right) .
$$

Note that $c_{i}$ is independent of $\gamma_{i}$. Then

$$
\mathbb{E}_{\alpha}\left[\left|X_{k, N}-U_{k, N}\right|\right] \leq\left|\alpha\left(t_{k+1}\right)-\alpha\left(t_{k}\right)\right| \mathbb{E}_{\alpha}\left[\left|\sum_{i=1}^{\infty} \gamma_{i} \frac{d}{d u}\left(C_{u} \Gamma_{i}^{-1 / u}\right)\left(c_{i}\right) \mathbf{1}_{\left[0, t_{k+1}\right]}\left(V_{i}\right)\right|\right.
$$

Let $p=1+\frac{\alpha^{*}}{2} \in\left(\alpha^{*}, 2\right)$. We use the fact that $u \mapsto C_{u}$ is uniformly bounded which implies that there exists $K>0$ such that $\left|\frac{d}{d u}\left(C_{u} \Gamma_{i}^{-1 / u}\right)\left(c_{i}\right)\right| \leq K\left(1+\left|\log \Gamma_{i}\right|\right)\left(\Gamma_{i}^{-1 / \alpha^{*}}+\Gamma_{i}^{-1 / \alpha_{*}}\right)$ to obtain

$$
\begin{aligned}
\mathbb{E}_{\alpha}\left[\left|\sum_{i=1}^{\infty} \gamma_{i} \frac{d}{d u}\left(C_{u} \Gamma_{i}^{-1 / u}\right)\left(c_{i}\right) \mathbf{1}_{\left[0, t_{k+1}\right]}\left(V_{i}\right)\right|\right] & \leq K \mathbb{E}_{\alpha}\left[\left(1+\left|\log \Gamma_{1}\right|\right)\left(\Gamma_{1}^{-1 / \alpha^{*}}+\Gamma_{1}^{-1 / \alpha_{*}}\right)\right] \\
& +K \mathbb{E}_{\alpha}\left[\left|\sum_{i=2}^{\infty} \gamma_{i} \frac{d}{d u}\left(C_{u} \Gamma_{i}^{-1 / u}\right)\left(c_{i}\right) \mathbf{1}_{\left[0, t_{k+1}\right]}\left(V_{i}\right)\right|\right] .
\end{aligned}
$$

Moreover, since $c_{i}$ is independent of $\gamma_{i}$, Theorem 2 of Von Bahr and Essen (1965) entails

$$
\begin{aligned}
\mathbb{E}_{\alpha}\left[\left|\sum_{i=1}^{\infty} \gamma_{i} \frac{d}{d u}\left(C_{u} \Gamma_{i}^{-1 / u}\right)\left(c_{i}\right) \mathbf{1}_{\left[0, t_{k+1}\right]}\left(V_{i}\right)\right|\right] & \leq K \mathbb{E}_{\alpha}\left[\left(1+\left|\log \Gamma_{1}\right|\right)\left(\Gamma_{1}^{-1 / \alpha^{*}}+\Gamma_{1}^{-1 / \alpha_{*}}\right)\right] \\
& +K \sum_{i=2}^{\infty} \mathbb{E}_{\alpha}\left[\left|\frac{d}{d u}\left(C_{u} \Gamma_{i}^{-1 / u}\right)\left(c_{i}\right)\right|^{p}\right]^{1 / p}
\end{aligned}
$$

whereby

$$
\begin{aligned}
\mathbb{E}_{\alpha}\left[\left|\sum_{i=1}^{\infty} \gamma_{i} \frac{d}{d u}\left(C_{u} \Gamma_{i}^{-1 / u}\right)\left(c_{i}\right) \mathbf{1}_{\left[0, t_{k+1}\right]}\left(V_{i}\right)\right|\right] & \leq K \mathbb{E}_{\alpha}\left[\left(1+\left|\log \Gamma_{1}\right|\right)\left(\Gamma_{1}^{-1 / \alpha^{*}}+\Gamma_{1}^{-1 / \alpha_{*}}\right)\right] \\
& +K \sum_{i=2}^{\infty} \mathbb{E}_{\alpha}\left[\left|\left(1+\left|\log \Gamma_{i}\right|\right)\left(\Gamma_{i}^{-1 / \alpha^{*}}+\Gamma_{i}^{-1 / \alpha_{*}}\right)\right|^{p}\right]^{1 / p}
\end{aligned}
$$


which is finite. Gathering all these inequalities, we obtain

$$
\mathbb{E}_{\alpha}\left[\left|X_{k, N}-U_{k, N}\right|\right] \leq K^{*}\left(\left|\alpha\left(t_{k+1}\right)-\alpha\left(t_{k}\right)\right| \leq \frac{K^{*} M}{N}\right.
$$

for some $K^{*}>0$. We derive from this last inequality and (31) that $N^{\left(1-\frac{1}{\alpha_{*}}\right)} \eta_{\mathbb{E}_{\alpha}}\left[|\log | \frac{U_{k, N}}{X_{k, N}}||^{2}\right]$ is uniformly bounded (in $k$ and $N$ ), and by (30), $N^{\left(1-\frac{1}{\alpha_{*}}\right) \eta} \mathbb{E}_{\alpha}\left[\left|\hat{h}_{N}(t)-\hat{h}_{N}^{U}(t)\right|^{2}\right]$ is uniformly bounded in $t \in[a, b], \alpha \in \Theta_{1}$ and $N$, that is $(26)$.

Proof of Lemma 8 Let us control $\mathbb{P}_{\alpha}\left(\left|X_{k, N}\right|>y\right)$ for any $y>1$. We write $c_{k, N}=$ $\min _{x \in\left[t_{k}, t_{k+1}\right]} \alpha(x)$. Using the truncation inequality (Loeve 1977, p. 209), one easily computes

$$
\begin{aligned}
\mathbb{P}_{\alpha}\left(\left|X_{k, N}\right|>y\right) & \leq y \int_{0}^{1 / y}\left(1-e^{-\int_{t_{k}}^{t_{k+1}}|\theta|^{\alpha(u)} d u}\right) d \theta \\
& \leq y \int_{0}^{1 / y} \int_{t_{k}}^{1 / t_{k+1}}|\theta|^{\alpha(u)} d u d \theta \\
& \leq y \int_{0}^{1 / y} \frac{|\theta|^{c_{k, N}}}{N} d \theta \\
& \leq \frac{1}{2 N y^{c_{k, N}}} .
\end{aligned}
$$

This leads to

$$
\begin{aligned}
\mathbb{E}_{\alpha}\left[N^{h(t)}\left|X_{k, N}\right|\right] & \leq 1+\frac{N^{h(t) c_{k, N}-1}}{2} \int_{1}^{+\infty} \frac{d y}{y^{c_{k, N}}} \\
& \leq 1+\left(\int_{1}^{+\infty} \frac{d y}{y^{\alpha_{*}}}\right) N^{\frac{c_{k, N}}{\alpha(t)}-1} .
\end{aligned}
$$

Thanks to the inequality $\left|\frac{c_{k, N}}{\alpha(t)}-1\right| \leq \frac{2\left\|\alpha^{\prime}\right\|_{\infty} n(N)}{N \alpha_{*}}$ whenever $k \in\left\{\lfloor N t\rfloor-\frac{n(N)}{2}, \ldots,\lfloor N t\rfloor+\right.$ $\left.\frac{n(N)}{2}-1\right\}$, we obtain

$$
\mathbb{E}_{\alpha}\left[N^{h(t)}\left|X_{k, N}\right|\right] \leq 1+\left(\int_{1}^{+\infty} \frac{d y}{y^{\alpha_{*}}}\right) \sup _{N} e^{\frac{2 M n(N) \log N}{N}}
$$

which is finite under the assumptions of the Lemma. This being true for every $N \geq 1, t \in[a, b]$, $\alpha \in \Theta_{1}$, and $k \in\left\{\lfloor N t\rfloor-\frac{n(N)}{2}, \ldots,\lfloor N t\rfloor+\frac{n(N)}{2}-1\right\},(27)$ holds.

In order to prove (28), notice that $W_{N}(t)=\sqrt{n(N)} \mu(\alpha(t))-\frac{1}{\sqrt{n(N)}} \sum_{k=\lfloor N t\rfloor-\frac{n(N)}{2}}^{\lfloor N t\rfloor+\frac{n(N)}{2}-1} \log \left(\left|N^{h(t)} X_{k, N}\right|\right)$. 
We use the following inequality for $t \in[a, b]$

$$
\begin{aligned}
e^{2\left|W_{N}(t)\right|} & \leq e^{2 W_{N}(t)}+e^{-2 W_{N}(t)} \\
& =e^{2 \sqrt{n(N)} \mu(\alpha(t))} \prod_{k=\lfloor N t\rfloor-\frac{n(N)}{2}}^{\lfloor N t\rfloor+\frac{n(N)}{2}-1} \frac{1}{\left|N^{h(t)} X_{k, N}\right|^{\frac{2}{\sqrt{n(N)}}}}+e^{-2 \sqrt{n(N)} \mu(\alpha(t))} \prod_{k=\lfloor N t\rfloor-\frac{n(N)}{2}}^{\lfloor N t\rfloor+\frac{n(N)}{2}-1}\left|N^{h(t)} X_{k, N}\right| \frac{2}{\sqrt{n(N)}}
\end{aligned}
$$

Taking the expectation, the independence of the variables $X_{k, N}$ and the Hölder inequality for $\sqrt{n(N)} \geq 16$ lead to

$$
\begin{aligned}
& \mathbb{E}_{\alpha}\left[e^{2\left|W_{N}(t)\right|}\right] \leq\left(K^{*}\right)^{\sqrt{n(N)}}\left(\prod_{k=\lfloor N t\rfloor-\frac{n(N)}{2}}^{\lfloor N t\rfloor+\frac{n(N)}{2}-1} \mathbb{E}_{\alpha}\left[\frac{1}{\left|N^{h(t)} Z_{k, N}\right| \frac{2}{\sqrt{n(N)}}}\right]+\prod_{k=\lfloor N t\rfloor-\frac{n(N)}{2}}^{\lfloor N t\rfloor+\frac{n(N)}{2}-1} \mathbb{E}_{\alpha}\left[\left|N^{h(t)} Z_{k, N}\right|^{\left.\frac{2}{\sqrt{n(N)}}\right]}\right]\right) \\
& \leq\left(K^{*}\right) \sqrt{n(N)}\left(\prod_{k=\lfloor N t\rfloor-\frac{n(N)}{2}}^{\lfloor N t\rfloor+\frac{n(N)}{2}-1} \mathbb{E}_{\alpha}\left[\left|N^{h(t)} Z_{k, N}\right|^{\left.-\frac{1}{8}\right]^{\frac{16}{\sqrt{n(N)}}}+} \prod_{k=\lfloor N t\rfloor-\frac{n(N)}{2}}^{\lfloor N t\rfloor+\frac{n(N)}{2}-1} \mathbb{E}_{\alpha}\left[\left|N^{h(t)} Z_{k, N}\right|\right]^{\frac{2}{\sqrt{n(N)}}}\right)\right. \\
& =\left(K^{*}\right)^{\sqrt{n(N)}} \prod_{k=\lfloor N t\rfloor-\frac{n(N)}{2}}^{\lfloor N t\rfloor+\frac{n(N)}{2}-1} N^{\frac{2\left(h\left(t_{k}\right)-h(t)\right)}{\sqrt{n(N)}}} \mathbb{E}_{\alpha}\left[\left|N^{h\left(t_{k}\right)} Z_{k, N}\right|^{-\frac{1}{8}}\right]^{\frac{16}{\sqrt{n(N)}}} \\
& \left.+\left(K^{*}\right)^{\sqrt{n(N)}} \prod_{k=\lfloor N t\rfloor-\frac{n(N)}{2}}^{\lfloor N t\rfloor+\frac{n(N)}{2}-1} \mathbb{E}_{\alpha}\left[\left|N^{h(t)} Z_{k, N}\right|\right]^{\frac{2}{\sqrt{n(N)}}}\right)
\end{aligned}
$$

where $K^{*}$ is some positive constant. Thanks to (24), (27), and the inequality $\left|h\left(t_{k}\right)-h(t)\right| \leq$ $\frac{M n(N)}{N}$ whenever $k \in\left\{\lfloor N t\rfloor-\frac{n(N)}{2}, \ldots,\lfloor N t\rfloor+\frac{n(N)}{2}-1\right\}$, we obtain

$$
\begin{aligned}
\mathbb{E}_{\alpha}\left[e^{2\left|W_{N}(t)\right|}\right] & \leq\left(K^{*}\right)^{\sqrt{n(N)}} e^{\frac{2 M(n(N))^{3 / 2} \log N}{N}}+\left(K^{*}\right)^{\sqrt{n(N)}} \\
& \leq\left(K^{*}\right)^{\sqrt{n(N)}}\left(\sup _{N} e^{\frac{2 M n(N) \log N}{N}}\right) \sqrt{n(N)}+\left(K^{*}\right)^{\sqrt{n(N)}}
\end{aligned}
$$

which implies (28).

Proof of theorem 1 If $\alpha \in \Theta_{0}$, we can rewrite for all $t \in[a, b], h(t)=h=\frac{1}{\alpha}$. Let $W_{N}(t)$ be $\xi_{N}\left(\hat{h}_{N}(t)-h\right)$ and recall that

$$
\begin{aligned}
T_{N} & =\frac{1}{\hat{\sigma}^{2}\left(t_{1}\right)(b-a)} \int_{a}^{b}\left|W_{N}(t)-W_{N}\left(t_{0}\right)\right|^{2} d t \\
& =\frac{1}{\hat{\sigma}^{2}\left(t_{1}\right)(b-a)} \int_{a}^{b}\left|W_{N}(t)\right|^{2} d t-2 \frac{W_{N}\left(t_{0}\right)}{\hat{\sigma}^{2}\left(t_{1}\right)(b-a)} \int_{a}^{b} W_{N}(t) d t+\frac{1}{\hat{\sigma}^{2}\left(t_{1}\right)}\left|W_{N}\left(t_{0}\right)\right|^{2} .
\end{aligned}
$$


In order to compute the Kolmogorov's distance between $P_{N}$ and $P_{1+\chi^{2}}$, we introduce the three terms in the last equality:

$$
\begin{aligned}
& W_{1}^{N}:=\frac{1}{\hat{\sigma}^{2}\left(t_{1}\right)(b-a)} \int_{a}^{b}\left|W_{N}(t)\right|^{2} d t-1, \\
& W_{2}^{N}:=-2 \frac{W_{N}\left(t_{0}\right)}{\hat{\sigma}^{2}\left(t_{1}\right)(b-a)} \int_{a}^{b} W_{N}(t) d t \\
& W_{3}^{N}:=\frac{1}{\hat{\sigma}^{2}\left(t_{1}\right)}\left|W_{N}\left(t_{0}\right)\right|^{2} .
\end{aligned}
$$

Let $x \in \mathbb{R}, \alpha \in \Theta_{0}$ and $Z \sim \mathcal{N}(0,1)$. It is easy to see that

$$
\begin{aligned}
\left|\mathbb{P}_{\alpha}\left(T_{N} \leq x\right)-\mathbb{P}_{\alpha}\left(1+|Z|^{2} \leq x\right)\right| & \leq\left|\mathbb{P}_{\alpha}\left(T_{N}-1 \leq x-1\right)-\mathbb{P}_{\alpha}\left(W_{3}^{N} \leq x-1\right)\right| \\
& +\left|\mathbb{P}_{\alpha}\left(W_{3}^{N} \leq x-1\right)-\mathbb{P}_{\alpha}\left(|Z|^{2} \leq x-1\right)\right| \\
& \leq \sup _{\alpha \in \Theta_{0}} \sup _{x \in \mathbb{R}}\left|\mathbb{P}_{\alpha}\left(T_{N}-1 \leq x\right)-\mathbb{P}_{\alpha}\left(W_{3}^{N} \leq x\right)\right| \\
& +\sup _{\alpha \in \Theta_{0}} \sup _{x \geq 0}\left|\mathbb{P}_{\alpha}\left(\frac{\left|W_{N}\left(t_{0}\right)\right|}{\sqrt{\hat{\sigma}^{2}\left(t_{1}\right)}} \leq x\right)-\mathbb{P}_{\alpha}(|Z| \leq x)\right| .
\end{aligned}
$$

We first consider (33): since $\alpha \in \Theta_{0}$ and $\left(t_{0}-\frac{n(N)}{2 N}, t_{0}+\frac{n(N)}{2 N}\right) \cap\left(t_{1}-\frac{n(N)}{2 N}, t_{1}+\frac{n(N)}{2 N}\right)=\varnothing$, $W_{N}\left(t_{0}\right)$ and $\hat{\sigma}^{2}\left(t_{1}\right)$ are two independent random variables. Write $F_{\alpha}^{N}$ for the distribution of $\frac{\sqrt{\hat{\sigma}^{2}\left(t_{1}\right)}}{\sqrt{\sigma^{2}(\alpha)}}$ :

$$
\mathbb{P}_{\alpha}\left(\frac{\left|W_{N}\left(t_{0}\right)\right|}{\sqrt{\hat{\sigma}^{2}\left(t_{1}\right)}} \leq x\right)=\int_{\mathbb{R}} \mathbb{P}_{\alpha}\left(\frac{\left|W_{N}\left(t_{0}\right)\right|}{\sqrt{\sigma^{2}(\alpha)}} \leq x y\right) d F_{\alpha}^{N}(y)
$$

and

$$
\begin{aligned}
\mathbb{P}_{\alpha}\left(\frac{\left|W_{N}\left(t_{0}\right)\right|}{\sqrt{\hat{\sigma}^{2}\left(t_{1}\right)}} \leq x\right)-\mathbb{P}_{\alpha}(|Z| \leq x) & =\int_{\mathbb{R}}\left[\mathbb{P}_{\alpha}\left(\frac{\left|W_{N}\left(t_{0}\right)\right|}{\sqrt{\sigma^{2}(\alpha)}} \leq x y\right)-\mathbb{P}_{\alpha}(|Z| \leq x y)\right] d F_{\alpha}^{N}(y) \\
& +\int_{\mathbb{R}}\left[\mathbb{P}_{\alpha}(|Z| \leq x y)-\mathbb{P}_{\alpha}(|Z| \leq x)\right] d F_{\alpha}^{N}(y) .
\end{aligned}
$$

Let $K>0$ be an absolute constant such that $\left|\mathbb{P}_{\alpha}(|Z| \leq x y)-\mathbb{P}_{\alpha}(|Z| \leq x)\right| \leq K|y-1|$. The constant $K$ may change from line to line in the following inequalitites. Using successively the facts that $\alpha \mapsto\left(\sigma^{2}(\alpha)\right)^{-1}$ is bounded, $x \mapsto \sqrt{\sigma^{2}(1 / x)}$ has a bounded derivative function and (12), we obtain

$$
\begin{aligned}
\sup _{x \geq 0} \int_{\mathbb{R}}\left|\mathbb{P}_{\alpha}(|Z| \leq x y)-\mathbb{P}_{\alpha}(|Z| \leq x)\right| d F_{\alpha}^{N}(y) & \leq K \mathbb{E}_{\alpha}\left[\left|\frac{\sqrt{\hat{\sigma}^{2}\left(t_{1}\right)}}{\sqrt{\sigma^{2}(\alpha)}}-1\right|\right] \\
& \leq K \mathbb{E}_{\alpha}\left[\left|\sqrt{\hat{\sigma}^{2}\left(t_{1}\right)}-\sqrt{\sigma^{2}(\alpha)}\right|\right] \\
& \leq K \mathbb{E}_{\alpha}\left[\left|\hat{h}_{N}\left(t_{1}\right)-h\right|\right] \\
& \leq K \frac{\sqrt{\sigma^{2}(\alpha)}}{\sqrt{n(N)}\left(\Gamma^{\prime}(1)+\log N\right)}
\end{aligned}
$$


This provides the existence of $K^{*}>0$ such that

$$
\sup _{\alpha \in \Theta_{0}} \sup _{x \geq 0} \int_{\mathbb{R}}\left|\mathbb{P}_{\alpha}(|Z| \leq x y)-\mathbb{P}_{\alpha}(|Z| \leq x)\right| d F_{\alpha}^{N}(y) \leq \frac{K^{*}}{\xi_{N}} .
$$

Now we get from (8) that $\frac{W_{N}\left(t_{0}\right)}{\sqrt{\sigma^{2}(\alpha)}}=\frac{\xi_{N}}{\sqrt{\sigma^{2}(\alpha)}}\left(\hat{h}_{N}\left(t_{0}\right)-h\right)=\frac{1}{\sqrt{n(N)}} \sum_{k=\left\lfloor N t_{0}\right\rfloor-\frac{n(N)}{2}}^{\left\lfloor t_{0}\right\rfloor+\frac{n(N)}{2}-1}\left(\frac{\mu(\alpha)-\log \left|N^{h} X_{k, N}\right|}{\sqrt{\sigma^{2}(\alpha)}}\right)$ where the variables $X_{k, N}$ are independent under $H_{0}$, so the Berry-Esseen inequality (see e.g. Petrov 1995, Theorem 5.5) gives the existence of an absolute constante $A$ such that

$$
\left|\mathbb{P}_{\alpha}\left(\frac{\left|W_{N}\left(t_{0}\right)\right|}{\sqrt{\sigma^{2}(\alpha)}} \leq x y\right)-\mathbb{P}_{\alpha}(|Z| \leq x y)\right| \leq A \frac{\mathbb{E}_{\alpha}\left[\left.|\mu(\alpha)-\log | W\right|^{3}\right]}{\sigma^{3}(\alpha) \sqrt{n(N)}}
$$

where $W \sim S_{\alpha}(1,0,0)$, and thanks to $(23)$,

$$
\sup _{\alpha \in \Theta_{0}} \sup _{x \in \mathbb{R}} \int_{\mathbb{R}}\left|\mathbb{P}_{\alpha}\left(\frac{\left|W_{N}\left(t_{0}\right)\right|}{\sqrt{\sigma^{2}(\alpha)}} \leq x y\right)-\mathbb{P}_{\alpha}(|Z| \leq x y)\right| d F_{\alpha}^{N}(y) \leq \frac{K^{*}}{\sqrt{n(N)}}
$$

Combining (34) and (35), we obtain an upper bound for (33) :

$$
\sup _{\alpha \in \Theta_{0}} \sup _{x \geq 0}\left|\mathbb{P}_{\alpha}\left(\frac{\left|W_{N}\left(t_{0}\right)\right|}{\sqrt{\hat{\sigma}^{2}\left(t_{1}\right)}} \leq x\right)-\mathbb{P}_{\alpha}(|Z| \leq x)\right| \leq K^{*}\left(\frac{1}{\xi_{N}}+\frac{1}{\sqrt{n(N)}}\right) .
$$

The second part of the proof deals with the term (32). Let $G_{N}$ be the cumulative distribution function of $W_{3}^{N}$, let $g_{N}(t)=\mathbb{E}_{\alpha}\left[e^{i t W_{3}^{N}}\right]$ and let $f_{N}(t)=\mathbb{E}_{\alpha}\left[e^{i t\left(T_{N}-1\right)}\right]$. Set $T>0$. Theorem 5.3 of Petrov (1995) leads to

$$
\begin{aligned}
\sup _{x \in \mathbb{R}}\left|\mathbb{P}_{\alpha}\left(T_{N}-1 \leq x\right)-\mathbb{P}_{\alpha}\left(W_{3}^{N} \leq x\right)\right| & \leq \frac{1}{\pi} \int_{-T}^{T}\left|\frac{f_{N}(t)-g_{N}(t)}{t}\right|\left(1-\frac{|t|}{T}\right) d t \\
& +\frac{T}{\pi} \sup _{x \in \mathbb{R}} \int_{|y| \leq \frac{C}{T}}\left|G_{N}(x+y)-G_{N}(x)\right| d y
\end{aligned}
$$

where $C$ is a positive constant which is not depending on $T$. From (36), we know that

$$
\begin{aligned}
\left|G_{N}(x+y)-G_{N}(x)\right| & \leq 2 K^{*}\left(\frac{1}{\xi_{N}}+\frac{1}{\sqrt{n(N)}}\right)+\left|\mathbb{P}_{\alpha}(|Z| \leq \sqrt{x+y})-\mathbb{P}_{\alpha}(|Z| \leq \sqrt{x})\right| \\
& \leq 2 K^{*}\left(\frac{1}{\xi_{N}}+\frac{1}{\sqrt{n(N)}}\right)+K \sqrt{|y|}
\end{aligned}
$$

where $K$ is an absolute constant. This gives an upper bound for (38): 


$$
\frac{T}{\pi} \sup _{\alpha \in \Theta_{0}} \sup _{x \in \mathbb{R}} \int_{|y| \leq \frac{C}{T}}\left|G_{N}(x+y)-G_{N}(x)\right| d y \leq K^{*}\left(\frac{1}{\xi_{N}}+\frac{1}{\sqrt{n(N)}}\right)+\frac{K}{\sqrt{T}} .
$$

Let us control (37). The inequality

$$
\begin{aligned}
\left|\frac{f_{N}(t)-g_{N}(t)}{t}\right| & =\left|\mathbb{E}_{\alpha}\left[\frac{e^{i t\left(W_{1}^{N}+W_{2}^{N}\right)}-1}{t} e^{i t W_{3}^{N}}\right]\right| \\
& \leq 2 \mathbb{E}_{\alpha}\left[\left|W_{1}^{N}+W_{2}^{N}\right|\right]
\end{aligned}
$$

yields

$$
\frac{1}{\pi} \int_{-T}^{T}\left|\frac{f_{N}(t)-g_{N}(t)}{t}\right|\left(1-\frac{|t|}{T}\right) d t \leq \frac{2 T}{\pi} \mathbb{E}_{\alpha}\left[\left|W_{1}^{N}+W_{2}^{N}\right|\right] .
$$

Gathering (36), (39), (37) and (40), we obtain an upper bound for the Kolmogorov's distance: we may find a constant $K^{*}$ such that for any $T>0$,

$$
\sup _{\alpha \in \Theta_{0}} \kappa\left(P_{N}^{\alpha}, P_{1+\chi^{2}}\right) \leq K^{*}\left(\frac{1}{\xi_{N}}+\frac{1}{\sqrt{n(N)}}+\frac{1}{\sqrt{T}}+T \sup _{\alpha \in \Theta_{0}} \mathbb{E}_{\alpha}\left[\left|W_{1}^{N}+W_{2}^{N}\right|\right]\right) .
$$

Minimizing (in $T$ ) the right term, we deduce that

$$
\sup _{\alpha \in \Theta_{0}} \kappa\left(P_{N}^{\alpha}, P_{1+\chi^{2}}\right) \leq K^{*}\left(\frac{1}{\xi_{N}}+\frac{1}{\sqrt{n(N)}}+\left(\sup _{\alpha \in \Theta_{0}} \mathbb{E}_{\alpha}\left[\left|W_{1}^{N}+W_{2}^{N}\right|\right]\right)^{1 / 3}\right)
$$

for some constant $K^{*}$. We finish the proof giving an upper bound for $\sup _{\alpha \in \Theta_{0}} \mathbb{E}_{\alpha}\left[\left|W_{1}^{N}+W_{2}^{N}\right|\right]$. Writing

$$
W_{1}^{N}=\frac{\sigma^{2}(\alpha)}{\hat{\sigma}^{2}\left(t_{1}\right)}\left[\frac{1}{b-a} \int_{a}^{b}\left|\frac{W_{N}(t)}{\sqrt{\sigma^{2}(\alpha)}}\right|^{2} d t-1\right]+\left[\frac{\sigma^{2}(\alpha)}{\hat{\sigma}^{2}\left(t_{1}\right)}-1\right],
$$

and using the boundness of the function $\sigma^{2}$, we obtain

$$
\begin{aligned}
\mathbb{E}_{\alpha}\left[\left|W_{1}^{N}\right|\right] & \leq K^{*} \mathbb{E}_{\alpha}\left[\left.\left|\frac{1}{b-a} \int_{a}^{b}\right| \frac{W_{N}(t)}{\sqrt{\sigma^{2}(\alpha)}}\right|^{2} d t-1 \mid\right]+K^{*} \mathbb{E}_{\alpha}\left[\left|\hat{h}_{N}\left(t_{1}\right)-h\right|\right] \\
& \leq K^{*} \mathbb{E}_{\alpha}\left[\left.\left|\frac{1}{b-a} \int_{a}^{b}\right| \frac{W_{N}(t)}{\sqrt{\sigma^{2}(\alpha)}}\right|^{2} d t-1 \mid\right]+\frac{K^{*}}{\xi_{N}}
\end{aligned}
$$

for some constant $K^{*}$. Thanks to (12),

$$
\mathbb{E}_{\alpha}\left[\left.\left|\frac{1}{b-a} \int_{a}^{b}\right| \frac{W_{N}(t)}{\sqrt{\sigma^{2}(\alpha)}}\right|^{2} d t-1 \mid\right] \leq \frac{4}{N(b-a)}+\mathbb{E}_{\alpha}\left[\left.\left|\frac{1}{b-a} \int_{\frac{\lfloor N a\rfloor+1}{N}}^{\frac{\lfloor N b\rfloor}{N}}\right| \frac{W_{N}(t)}{\sqrt{\sigma^{2}(\alpha)}}\right|^{2} d t-1 \mid\right] .
$$


Making explicit $W_{N}(t)$, one can computes

$\int_{\frac{\lfloor N a\rfloor+1}{N}}^{\frac{\lfloor N b\rfloor}{N}}\left|\frac{W_{N}(t)}{\sqrt{\sigma^{2}(\alpha)}}\right|^{2} d t=\frac{1}{N} \sum_{j=\lfloor N a\rfloor+1}^{\lfloor N b\rfloor-1}\left|\frac{W_{N}\left(\frac{j}{N}\right)}{\sqrt{\sigma^{2}(\alpha)}}\right|^{2}=\frac{1}{N n(N)} \sum_{j=\lfloor N a\rfloor+1}^{\lfloor N b\rfloor-1}\left|\sum_{k=j-\frac{n(N)}{2}}^{j+\frac{n(N)}{2}-1} \frac{\mu(\alpha)-\log \left|N^{h} X_{k, N}\right|}{\sqrt{\sigma^{2}(\alpha)}}\right|^{2}$.

If $\alpha \in \Theta_{0}$, recall that the variables $U_{k}:=\frac{\mu(\alpha)-\log \left|N^{h} X_{k, N}\right|}{\sqrt{\sigma^{2}(\alpha)}}$ are standard independent random variables. Consequently we can rewrite the last sum

$$
\begin{aligned}
\frac{1}{N n(N)} \sum_{j=\lfloor N a\rfloor+1}^{\lfloor N b\rfloor-1}\left|\sum_{k=j-\frac{n(N)}{2}}^{j+\frac{n(N)}{2}-1} U_{k}\right|^{2}=\frac{1}{N n(N)} \sum_{j=\lfloor N a\rfloor+1}^{\lfloor N b\rfloor-1} \sum_{k=j-\frac{n(N)}{2}}^{j+\frac{n(N)}{2}-1} U_{k}^{2}+\frac{2}{N n(N)} \sum_{j=\lfloor N a\rfloor+1}^{\lfloor N b\rfloor-1} \sum_{k=j-\frac{n(N)}{2}}^{j+\frac{n(N)}{2}-1} \sum_{k^{\prime}=k+1}^{j+\frac{n(N)}{2}-1} U_{k} U_{k^{\prime}} \\
=: \Sigma_{1}+\Sigma_{2},
\end{aligned}
$$

whereby (42) and (43) lead to

$$
\mathbb{E}_{\alpha}\left[\left|W_{1}^{N}\right|\right] \leq K^{*}\left(\frac{1}{\xi_{N}}+\frac{1}{N(b-a)}\right)+\mathbb{E}_{\alpha}\left[\left|\frac{1}{b-a} \Sigma_{1}-1\right|\right]+\mathbb{E}_{\alpha}\left[\left|\Sigma_{2}\right|\right]
$$

We are left with the task of determining an upper bound for $\mathbb{E}_{\alpha}\left[\left|\frac{1}{b-a} \Sigma_{1}-1\right|\right]$ and $\mathbb{E}_{\alpha}\left[\left|\Sigma_{2}\right|\right]$. For the first term $\Sigma_{1}$, we invert the two sums to get:

$$
\begin{aligned}
\Sigma_{1} & =\frac{1}{N n(N)}\left(\sum_{k=\lfloor N a\rfloor+1-\frac{n(N)}{2}}^{\lfloor N a\rfloor+\frac{n(N)}{2}-1}\left(k+\frac{n(N)}{2}-\lfloor N a\rfloor\right) U_{k}^{2}+\sum_{k=\lfloor N a\rfloor+\frac{n(N)}{2}}^{\lfloor N b\rfloor-1-\frac{n(N)}{2}} n(N) U_{k}^{2}\right) \\
& +\frac{1}{N n(N)} \sum_{k=\lfloor N b\rfloor-\frac{n(N)}{2}}^{\lfloor N b\rfloor-2+\frac{n(N)}{2}}\left(\lfloor N b\rfloor+\frac{n(N)}{2}-k-1\right) U_{k}^{2} .
\end{aligned}
$$

For the computation of $\mathbb{E}_{\alpha}\left[\left|\frac{1}{b-a} \Sigma_{1}-1\right|\right]$, notice first that

$\mathbb{E}_{\alpha}\left[\frac{1}{N n(N)}\left(\sum_{k=\lfloor N a\rfloor+1-\frac{n(N)}{2}}^{\lfloor N a\rfloor+\frac{n(N)}{2}-1}\left(k+\frac{n(N)}{2}-\lfloor N a\rfloor\right) U_{k}^{2}+\sum_{k=\lfloor N b\rfloor-\frac{n(N)}{2}}^{\lfloor N b\rfloor-2+\frac{n(N)}{2}}\left(\lfloor N b\rfloor+\frac{n(N)}{2}-k-1\right) U_{k}^{2}\right)\right] \leq \frac{2 n(N)}{N}$,

so we are reduced to consider

$$
\begin{aligned}
\frac{1}{N(b-a)} \sum_{k=\lfloor N a\rfloor+\frac{n(N)}{2}}^{\lfloor N b\rfloor-1-\frac{n(N)}{2}} U_{k}^{2}-1 & =\left(\frac{\lfloor N b\rfloor-\lfloor N a\rfloor-n(N)}{N}-1\right) \frac{1}{\lfloor N b\rfloor-\lfloor N a\rfloor-n(N)} \sum_{k=\lfloor N a\rfloor+\frac{n(N)}{2}}^{\lfloor N b\rfloor-1-\frac{n(N)}{2}} U_{k}^{2} \\
& +\frac{1}{\lfloor N b\rfloor-\lfloor N a\rfloor-n(N)} \sum_{k=\lfloor N a\rfloor+\frac{n(N)}{2}}^{\lfloor N b\rfloor-1-\frac{n(N)}{2}}\left(U_{k}^{2}-1\right) .
\end{aligned}
$$


Since $\left|\frac{\lfloor N b\rfloor-\lfloor N a\rfloor-n(N)}{N}-1\right| \leq \frac{n(N)+2}{N(b-a)}$,

$$
\mathbb{E}_{\alpha}\left[\left|\frac{1}{b-a} \Sigma_{1}-1\right|\right] \leq \frac{2 n(N)}{N(b-a)}+\sqrt{\frac{\operatorname{var}_{\alpha}\left(U_{1}^{2}\right)}{\lfloor N b\rfloor-\lfloor N a\rfloor-n(N)}} .
$$

For the second term $\Sigma_{2}$, we invert the two first sums:

$$
\begin{aligned}
\Sigma_{2} & =\frac{2}{N n(N)} \sum_{k=\lfloor N a\rfloor+1-\frac{n(N)}{2}}^{\lfloor N a\rfloor+\frac{n(N)}{2}-1} U_{k} \sum_{j=\lfloor N a\rfloor+1}^{k+\frac{n(N)}{2}} \sum_{k^{\prime}=k+1}^{j+\frac{n(N)}{2}} U_{k^{\prime}}+\frac{2}{N n(N)} \sum_{k=\lfloor N a\rfloor+\frac{n(N)}{2}}^{\lfloor N b\rfloor-1-\frac{n(N)}{2}} U_{k} \sum_{j=k-\frac{n(N)}{2}+1}^{k+\frac{n(N)}{2}} \sum_{k^{\prime}=k+1}^{j+\frac{n(N)}{2}} U_{k^{\prime}} \\
& +\frac{2}{N n(N)} \sum_{k=\lfloor N b\rfloor-\frac{n(N)}{2}} U_{k} \sum_{j=k-\frac{n(N)}{2}+1}^{\lfloor N b\rfloor-2+\frac{n(N)}{2}} \sum_{k^{\prime}=k+1}^{j N b\rfloor-1} U_{k^{\prime}} .
\end{aligned}
$$

We define $S_{k}$ to be $\sum_{j=\lfloor N a\rfloor+1}^{k+\frac{n(N)}{2}} \sum_{k^{\prime}=k+1}^{j+\frac{n(N)}{2}} U_{k^{\prime}}$ if $k \in\left\{\lfloor N a\rfloor+1-\frac{n(N)}{2}, \ldots,\lfloor N a\rfloor+\frac{n(N)}{2}-1\right\}$, $\sum_{j=k-\frac{n(N)}{2}+1}^{k+\frac{n(N)}{2}} \sum_{k^{\prime}=k+1}^{j+\frac{n(N)}{2}} U_{k^{\prime}}$ if $k \in\left\{\lfloor N a\rfloor+\frac{n(N)}{2}, \ldots,\lfloor N b\rfloor-1-\frac{n(N)}{2}\right\}$ and $\sum_{j=k-\frac{n(N)}{2}+1}^{\lfloor N b\rfloor-1} \sum_{k^{\prime}=k+1}^{j+\frac{n(N)}{2}} U_{k^{\prime}}$ if $k \in\left\{\lfloor N b\rfloor-\frac{n(N)}{2}, \ldots,\lfloor N b\rfloor-2+\frac{n(N)}{2}\right\}$, in order to rewrite

$$
\Sigma_{2}=\frac{2}{N n(N)} \sum_{k=\lfloor N a\rfloor+1-\frac{n(N)}{2}}^{\lfloor N b\rfloor-2+\frac{n(N)}{2}} U_{k} S_{k}
$$

$S_{k}$ is a sum of variables $U_{i}$ with indices greater than $k$ so $S_{k}$ is independent of $U_{k}$ and $\mathbb{E}_{\alpha}\left[U_{k} S_{k}\right]=0$. Moreover, $\mathbb{E}_{\alpha}\left[S_{k}^{2}\right] \leq n(N)^{3}$. Therefore it is easy to see that

$$
\begin{aligned}
\operatorname{var}_{\alpha}\left(\Sigma_{2}\right) & =\frac{4}{N^{2} n(N)^{2}} \sum_{k=\lfloor N a\rfloor+1-\frac{n(N)}{2}}^{\lfloor N b\rfloor-2+\frac{n(N)}{2}} \mathbb{E}_{\alpha}\left[U_{k}^{2}\right] \mathbb{E}_{\alpha}\left[S_{k}^{2}\right] \\
& \leq \frac{4}{N^{2} n(N)^{2}} \sum_{k=\lfloor N a\rfloor+1-\frac{n(N)}{2}}^{\lfloor N b\rfloor-2+\frac{n(N)}{2}} n(N)^{3} \\
& \leq \frac{4 n(N)(N+n(N))}{N^{2}} \\
& \leq \frac{8 n(N)}{N}
\end{aligned}
$$

Combining (44), (45) and (47), we obtain

$$
\sup _{\alpha \in \Theta_{0}} \mathbb{E}_{\alpha}\left[\left|W_{1}^{N}\right|\right] \leq K^{*}\left(\sqrt{\frac{n(N)}{N}}+\frac{1}{\xi_{N}}+\frac{1}{\sqrt{\lfloor N b\rfloor-\lfloor N a\rfloor-n(N)}}\right)
$$


Let us conclude the proof giving an upper bound for $\mathbb{E}_{\alpha}\left[\left|W_{2}^{N}\right|\right]$. Notice first that $\mathbb{E}_{\alpha}\left[W_{N}\left(t_{0}\right)\right]=$ $\mathbb{E}_{\alpha}\left[\int_{a}^{b} W_{N}(t) d t\right]=0$. Then, if $\alpha \in \Theta_{0}$,

$$
\mathbb{E}_{\alpha}\left[\left|W_{2}^{N}\right|\right] \leq K^{*} \sqrt{\operatorname{var}_{\alpha}\left(W_{N}\left(t_{0}\right)\right)} \sqrt{\operatorname{var}_{\alpha}\left(\int_{a}^{b} W_{N}(t) d t\right)}=K^{*} \sqrt{\sigma^{2}(\alpha)} \sqrt{\operatorname{var}_{\alpha}\left(\int_{a}^{b} W_{N}(t) d t\right)} .
$$

It remains to control $\operatorname{var}_{\alpha}\left(\int_{a}^{b} W_{N}(t) d t\right)$.

$$
\begin{aligned}
\int_{a}^{b} W_{N}(t) d t & =\int_{a}^{\frac{\lfloor N a\rfloor+1}{N}} W_{N}(t) d t+\int_{\frac{\lfloor N a\rfloor+1}{N}}^{\frac{\lfloor N b\rfloor}{N}} W_{N}(t) d t+\int_{\frac{\lfloor N b\rfloor}{N}}^{b} W_{N}(t) d t \\
& =\left(\frac{\lfloor N a\rfloor+1}{N}-a\right) W_{N}\left(\frac{\lfloor N a\rfloor}{N}\right)+\int_{\frac{\lfloor N a\rfloor+1}{N}}^{\frac{\lfloor N b\rfloor}{N}} W_{N}(t) d t+\left(b-\frac{\lfloor N b\rfloor}{N}\right) W_{N}\left(\frac{\lfloor N b\rfloor}{N}\right) \\
& =c_{N} \sum_{j=\lfloor N a\rfloor}^{\lfloor N b\rfloor} \sum_{k=j-\frac{n(N)}{2}}^{j+\frac{n(N)}{2}}-1
\end{aligned}
$$

where $U_{k}=\mu(\alpha)-\log \left(N^{h} X_{k, N}\right)$ and $\left|c_{N}\right| \leq \frac{2}{N \sqrt{n(N)}}$, so inverting again the sums as we have done for $\Sigma_{1}$,

$$
\begin{aligned}
\int_{a}^{b} W_{N}(t) d t & =c_{N}\left(\sum_{k=\lfloor N a\rfloor-\frac{n(N)}{2}}^{\lfloor N a\rfloor+\frac{n(N)}{2}-2}\left(k+\frac{n(N)}{2}-\lfloor N a\rfloor+1\right) U_{k}+\sum_{k=\lfloor N a\rfloor+\frac{n(N)}{2}-1}^{\lfloor N b\rfloor-\frac{n(N)}{2}} n(N) U_{k}\right. \\
& \left.+\sum_{k=\lfloor N b\rfloor+1-\frac{n(N)}{2}}^{\lfloor N b\rfloor-1+\frac{n(N)}{2}}\left(\lfloor N b\rfloor+\frac{n(N)}{2}-k\right) U_{k}\right) \\
& =\sum_{k=\lfloor N a\rfloor-\frac{n(N)}{2}}^{\lfloor N b\rfloor+\frac{n(N)}{2}-1} d_{k} U_{k}
\end{aligned}
$$

with $\left|d_{k}\right| \leq \frac{2 \sqrt{n(N)}}{N}$. Thus,

$$
\begin{aligned}
\operatorname{var}_{\alpha}\left(\int_{a}^{b} W_{N}(t) d t\right) & =\sigma^{2}(\alpha) \sum_{k=\lfloor N a\rfloor-\frac{n(N)}{2}}^{\lfloor N b\rfloor+\frac{n(N)}{2}-1} d_{k}^{2} \\
& \leq \sigma^{2}(\alpha) \frac{4 n(N)}{N^{2}}(\lfloor N b\rfloor-\lfloor N a\rfloor+n(N)) \\
& \leq K^{*} \frac{n(N)}{N}
\end{aligned}
$$

We obtain then

$$
\sup _{\alpha \in \Theta_{0}} \mathbb{E}_{\alpha}\left[\left|W_{2}^{N}\right|\right] \leq K^{*} \sqrt{\frac{n(N)}{N}}
$$


We thus derive (15) from (41), (48) and (49)

Proof of THEOREM 2

Let $\chi$ be a random variable distributed as $\sigma_{\chi} Z$ where $Z \sim \mathcal{N}(0,1)$ and $W_{N}$ be equal to

$$
W_{N}=\sigma_{\chi} \frac{\xi_{N}\left[\bar{h}\left(t_{0}\right)-\hat{h}_{N}^{U}\left(t_{0}\right)\right]}{\left(B_{N}\right)^{1 / 2}}
$$

where $B_{N}=\frac{1}{n(N)} \sum_{k=\left\lfloor N t_{0}\right\rfloor-\frac{n(N)}{2}}^{\left\lfloor N t_{0}\right\rfloor+\frac{n(N)}{2}-1} \sigma^{2}\left(\alpha\left(\frac{k}{N}\right)\right)$.

Fix $x \in \mathbb{R}$ and $\alpha \in \Theta_{1}$. It is easy to see that

$$
\begin{aligned}
\left|\mathbb{P}_{\alpha}\left(\chi_{N} \leq x\right)-\mathbb{P}_{\alpha}(\chi \leq x)\right| & =\left|\mathbb{P}_{\alpha}\left(\chi_{N} \leq x\right)-\mathbb{P}_{\alpha}\left(W_{N} \leq x\right)+\mathbb{P}_{\alpha}\left(W_{N} \leq x\right)-\mathbb{P}_{\alpha}(\chi \leq x)\right| \\
& \leq \sup _{x \in \mathbb{R}}\left|\mathbb{P}_{\alpha}\left(W_{N} \leq x\right)-\mathbb{P}_{\alpha}(\chi \leq x)\right| \\
& +\sup _{x \in \mathbb{R}}\left|\mathbb{P}_{\alpha}\left(\chi_{N} \leq x\right)-\mathbb{P}_{\alpha}\left(W_{N} \leq x\right)\right|
\end{aligned}
$$

The proof falls naturally into two steps. We first compute an upper bound for (51):

$$
\sup _{x \in \mathbb{R}}\left|\mathbb{P}_{\alpha}\left(W_{N} \leq x\right)-\mathbb{P}_{\alpha}(\chi \leq x)\right|=\sup _{x \in \mathbb{R}}\left|\mathbb{P}_{\alpha}\left(\frac{\xi_{N}\left[\bar{h}\left(t_{0}\right)-\hat{h}_{N}^{U}\left(t_{0}\right)\right]}{\left(B_{N}\right)^{1 / 2}} \leq x\right)-\mathbb{P}_{\alpha}(Z \leq x)\right|
$$

where $Z \sim \mathcal{N}(0,1)$. Since

$$
\frac{\xi_{N}\left[\bar{h}\left(t_{0}\right)-\hat{h}_{N}^{U}\left(t_{0}\right)\right]}{\left(B_{N}\right)^{1 / 2}}=\frac{\sum_{k=\left\lfloor N t_{0}\right\rfloor-\frac{n(N)}{2}}^{\left\lfloor N t_{0}\right\rfloor+\frac{n(N)}{2}-1}\left(\log \left|U_{k, N}\right|-\mathbb{E}_{\alpha}\left[\log \left|U_{k, N}\right|\right]\right)}{\left(\sum_{k=\left\lfloor N t_{0}\right\rfloor-\frac{n(N)}{2}}^{\left\lfloor N t_{0}\right\rfloor+\frac{n(N)}{2}-1} \operatorname{var}_{\alpha}\left(\log \left|U_{k, N}\right|\right)\right)^{1 / 2}}
$$

we can use Theorem 5.4 of Petrov (1995) to obtain the existence of an absolute constant $A>0$ such that

$$
\sup _{x \in \mathbb{R}}\left|\mathbb{P}_{\alpha}\left(W_{N} \leq x\right)-\mathbb{P}_{\alpha}(\chi \leq x)\right| \leq A \frac{\sum_{k=\left\lfloor N t_{0}\right\rfloor-\frac{n(N)}{2}}^{\left\lfloor N t_{0}\right\rfloor+\frac{n(N)}{2}-1} \mathbb{E}_{\alpha}\left[|\log | U_{k, N}\left|-\mathbb{E}_{\alpha}\left[\log \left|U_{k, N}\right|\right]\right|^{3}\right]}{\left(\sum_{k=\left\lfloor N t_{0}\right\rfloor-\frac{n(N)}{2}}^{\left\lfloor N t_{0}\right\rfloor+\frac{n(N)}{2}-1} \sigma^{2}\left(\alpha\left(\frac{k}{N}\right)\right)\right)^{3 / 2}} .
$$

Using Lemma 5 and the fact that $\inf _{t} \sigma^{2}(t)$ is positive, we obtain

$$
\sup _{x \in \mathbb{R}}\left|\mathbb{P}_{\alpha}\left(W_{N} \leq x\right)-\mathbb{P}_{\alpha}(\chi \leq x)\right| \leq K^{*} \frac{n(N)}{n(N)^{3 / 2}}
$$

for some constant $K^{*}$, whereby 


$$
\sup _{\alpha \in \Theta_{1}} \sup _{x \in \mathbb{R}}\left|\mathbb{P}_{\alpha}\left(W_{N} \leq x\right)-\mathbb{P}_{\alpha}(\chi \leq x)\right| \leq \frac{K^{*}}{\sqrt{n(N)}} .
$$

The second part of the proof deals with the term (52). Let $G_{N}$ be the cumulative distribution function of $W_{N}$, let $g_{N}(t)=\mathbb{E}_{\alpha}\left[e^{i t W_{N}}\right]$ and let $f_{N}(t)=\mathbb{E}_{\alpha}\left[e^{i t \chi_{N}}\right]$. Set $T>0$. Theorem 5.3 of Petrov (1995) leads to

$$
\begin{aligned}
\sup _{x \in \mathbb{R}}\left|\mathbb{P}_{\alpha}\left(\chi_{N} \leq x\right)-\mathbb{P}_{\alpha}\left(W_{N} \leq x\right)\right| & \leq \frac{1}{\pi} \int_{-T}^{T}\left|\frac{f_{N}(t)-g_{N}(t)}{t}\right|\left(1-\frac{|t|}{T}\right) d t \\
& +\frac{T}{\pi} \sup _{x \in \mathbb{R}} \int_{|y| \leq \frac{C}{T}}\left|G_{N}(x+y)-G_{N}(x)\right| d y
\end{aligned}
$$

where $C$ is a positive constant which is not depending on $T$. By (53) we get

$$
\left|G_{N}(x+y)-G_{N}(x)\right| \leq \frac{2 K^{*}}{\sqrt{n(N)}}+|G(x+y)-G(x)| \leq \frac{K^{*}}{\sqrt{n(N)}}+\frac{K|y|}{\sigma_{\chi}}
$$

where $K$ is an absolute constant. This provides an upper bound for (55):

$$
\frac{T}{\pi} \sup _{x \in \mathbb{R}} \int_{|y| \leq \frac{C}{T}}\left|G_{N}(x+y)-G_{N}(x)\right| d y \leq \frac{K^{*}}{\sqrt{n(N)}}+\frac{K}{\sigma_{\chi} T} .
$$

Let us control (54):

$$
\begin{aligned}
\left|\frac{f_{N}(t)-g_{N}(t)}{t}\right| & =\left|\mathbb{E}_{\alpha}\left[\frac{e^{i t\left(\chi_{N}-W_{N}\right)}-1}{t} e^{i t W_{N}}\right]\right| \\
& \leq 2 E_{\alpha}\left[\left|\chi_{N}-W_{N}\right|\right]
\end{aligned}
$$

SO

$$
\frac{1}{\pi} \int_{-T}^{T}\left|\frac{f_{N}(t)-g_{N}(t)}{t}\right|\left(1-\frac{|t|}{T}\right) d t \leq \frac{2 T}{\pi} \mathbb{E}_{\alpha}\left[\left|\chi_{N}-W_{N}\right|\right]
$$

We derive an upper bound for the Kolmogorov's distance from (53), (56) and (57): there exists $K^{*}>0$ such that for every $T>0$,

$$
\kappa\left(P_{\chi_{N}}, P_{\chi}\right) \leq K^{*}\left(\frac{1}{\sqrt{n(N)}}+\frac{1}{\sigma_{\chi} T}+T \sup _{\alpha \in \Theta_{1}} \mathbb{E}_{\alpha}\left[\left|\chi_{N}-W_{N}\right|\right]\right) .
$$

Minimizing (in $T$ ) the right term, we deduce that there exists $K^{*}>0$ such that

$$
\kappa\left(P_{\chi_{N}}, P_{\chi}\right) \leq K^{*}\left(\frac{1}{\sqrt{n(N)}}+\frac{\left(\sup _{\alpha \in \Theta_{1}} \mathbb{E}_{\alpha}\left[\left|\chi_{N}-W_{N}\right|\right]\right)^{1 / 2}}{\sqrt{\sigma_{\chi}}}\right) .
$$


We finish the proof giving an upper bound for $\sup _{\alpha \in \Theta_{1}} \mathbb{E}_{\alpha}\left[\left|\chi_{N}-W_{N}\right|\right]$. We use the equality $\int_{a}^{b}\left|\hat{h}_{N}^{U}(t)-\hat{h}_{N}^{U}\left(t_{0}\right)\right|^{2} d t=\int_{a}^{b}\left|\hat{h}_{N}^{U}(t)-\bar{h}(t)+\bar{h}(t)-\bar{h}\left(t_{0}\right)+\bar{h}\left(t_{0}\right)-\hat{h}_{N}^{U}\left(t_{0}\right)\right|^{2} d t$ and we expand the square to obtain seven terms for $\chi_{N}-W_{N}$ :

$$
\begin{aligned}
\frac{\hat{\sigma}^{2}\left(t_{1}\right)}{\xi_{N}}\left(\chi_{N}-W_{N}\right) & =\frac{1}{b-a}\left[\int_{a}^{b}\left|\hat{h}_{N}(t)-\hat{h}_{N}\left(t_{0}\right)\right|^{2} d t-\int_{a}^{b}\left|\hat{h}_{N}^{U}(t)-\hat{h}_{N}^{U}\left(t_{0}\right)\right|^{2} d t\right] \\
& +\frac{1}{b-a} \int_{a}^{b}\left|\hat{h}_{N}^{U}(t)-\bar{h}(t)\right|^{2} d t \\
& +\frac{1}{b-a}\left[\int_{a}^{b}\left|\bar{h}(t)-\bar{h}\left(t_{0}\right)\right|^{2} d t-\int_{a}^{b}\left|h(t)-h\left(t_{0}\right)\right|^{2} d t\right] \\
& +\left|\bar{h}\left(t_{0}\right)-\hat{h}_{N}^{U}\left(t_{0}\right)\right|^{2} \\
& +\frac{2}{b-a} \int_{a}^{b}\left(\hat{h}_{N}^{U}(t)-\bar{h}(t)\right)\left(\bar{h}(t)-\bar{h}\left(t_{0}\right)\right) d t \\
& +\frac{2\left[\bar{h}\left(t_{0}\right)-\hat{h}_{N}^{U}\left(t_{0}\right)\right]}{b-a} \int_{a}^{b}\left(\hat{h}_{N}^{U}(t)-\bar{h}(t)\right) d t \\
& +\frac{2\left[\bar{h}\left(t_{0}\right)-\hat{h}_{N}^{U}\left(t_{0}\right)\right]}{b-a} \int_{a}^{b}\left(\bar{h}(t)-\bar{h}\left(t_{0}\right)\right) d t-\frac{\hat{\sigma}^{2}\left(t_{1}\right)}{\xi_{N}} W_{N}
\end{aligned}
$$

The end of the proof consists in computing an upper bound for each term $Q_{j}$. Let $\eta$ be a fixed number in $\left(0, \frac{1}{2}\right)$.

\section{Upper bound for $Q_{1}$ Write}

$$
\begin{aligned}
Q_{1} & =\frac{1}{b-a} \int_{a}^{b}\left|\hat{h}_{N}(t)-\hat{h}_{N}^{U}(t)\right|^{2} d t+\left|\hat{h}_{N}^{U}\left(t_{0}\right)-\hat{h}_{N}\left(t_{0}\right)\right|^{2} \\
& +\frac{2}{b-a} \int_{a}^{b}\left(\hat{h}_{N}(t)-\hat{h}_{N}^{U}(t)\right)\left(\hat{h}_{N}^{U}(t)-\hat{h}_{N}^{U}\left(t_{0}\right)\right) d t \\
& +\frac{2}{b-a}\left(\hat{h}_{N}^{U}\left(t_{0}\right)-\hat{h}_{N}\left(t_{0}\right)\right) \int_{a}^{b}\left(\hat{h}_{N}(t)-\hat{h}_{N}^{U}(t)\right) d t \\
& +\frac{2}{b-a}\left(\hat{h}_{N}^{U}\left(t_{0}\right)-\hat{h}_{N}\left(t_{0}\right)\right) \int_{a}^{b}\left(\hat{h}_{N}^{U}(t)-\hat{h}_{N}^{U}\left(t_{0}\right)\right) d t
\end{aligned}
$$

Using the fact that $\sup \sup \sup \mathbb{E}_{\alpha}\left[\left|\hat{h}_{N}^{U}(t)\right|^{2}\right]<+\infty$, the Hölder inequality and (26) yield $N \quad \alpha \in \Theta_{1} t \in[a, b]$ 


$$
\mathbb{E}_{\alpha}\left[\left|Q_{1}\right|\right] \leq \frac{K_{\eta}^{*}}{N^{\left(1-\frac{1}{\alpha_{*}}\right) \frac{\eta}{2}}}
$$

for some positive constant $K_{\eta}^{*}$.

Upper bound for $Q_{2}$ and $Q_{4}$ Thanks to (25),

$$
\mathbb{E}_{\alpha}\left[\left|Q_{2}+Q_{4}\right|\right] \leq \frac{K}{\xi_{N}^{2}}
$$

Upper bound for $Q_{3}$ Since $|\bar{h}(t)-h(t)| \leq K^{*} \frac{n(N)}{N}$ and $\left(\frac{n(N)}{N}\right)^{2} \leq \frac{n(N)}{N}$ for $N$ large enough, it is not difficult to see that

$$
\mathbb{E}_{\alpha}\left[\left|Q_{3}\right|\right]=\left|Q_{3}\right| \leq K^{*} \frac{n(N)}{N} .
$$

Upper bound for $Q_{5} \quad$ Lemma 7 leads to

$$
\sup _{\alpha \in \Theta_{1}} \mathbb{E}_{\alpha}\left[\left|\frac{2}{b-a} \int_{a}^{\frac{\lfloor N a\rfloor+1}{N}}\left(\hat{h}_{N}^{U}(t)-\bar{h}(t)\right)\left(\bar{h}(t)-\bar{h}\left(t_{0}\right)\right) d t\right|\right] \leq \frac{K^{*}}{\xi_{N}}\left(\frac{\lfloor N a\rfloor+1}{N}-a\right) \leq \frac{K^{*}}{N \xi_{N}} .
$$

For the same reasons we get

$$
\sup _{\alpha \in \Theta_{1}} \mathbb{E}_{\alpha}\left[\left|\frac{2}{b-a} \int_{\frac{\mid N b}{N}}^{b}\left(\hat{h}_{N}^{U}(t)-\bar{h}(t)\right)\left(\bar{h}(t)-\bar{h}\left(t_{0}\right)\right) d t\right|\right] \leq \frac{K^{*}}{N \xi_{N}} .
$$

Thereby, defining $I_{a, b}$ as $\int_{\frac{\lfloor N a\rfloor+1}{N}}^{\frac{\lfloor N b\rfloor}{N}}\left(\hat{h}_{N}^{U}(t)-\bar{h}(t)\right)\left(\bar{h}(t)-\bar{h}\left(t_{0}\right)\right) d t$, we have

$$
I_{a, b}=\frac{1}{\sqrt{n(N)} \xi_{N}} \sum_{j=\lfloor N a\rfloor+1}^{\lfloor N b\rfloor-1} \sum_{k=j-\frac{n(N)}{2}}^{j+\frac{n(N)}{2}-1} W_{k, j}
$$

where $W_{k, j}=\left(\log \left|U_{k, N}\right|-\mathbb{E}_{\alpha}\left[\log \left|U_{k, N}\right|\right]\right) \int_{\frac{j}{N}}^{\frac{j+1}{N}}\left(\bar{h}(t)-\bar{h}\left(t_{0}\right)\right) d t$. We apply Lemma 4 in order to 
obtain

$$
\begin{array}{r}
I_{a, b}=\frac{1}{\sqrt{n(N) \xi_{N}}} \sum_{k=\lfloor N a\rfloor+1}^{\lfloor N a\rfloor+\frac{n(N)}{2}-1} V_{k, N} \int_{\frac{\lfloor N a\rfloor+1}{N}}^{\frac{k+1+\frac{n(N)}{2}}{N}}\left(\bar{h}(t)-\bar{h}\left(t_{0}\right)\right) d t \\
+\frac{1}{\sqrt{n(N) \xi_{N}}} \sum_{k=\lfloor N a\rfloor+\frac{n(N)}{2}} V_{k, N} \int_{\frac{k+1-\frac{n(N)}{2}}{N}}^{\frac{k+1+\frac{n(N)}{2}}{\int^{N}}}\left(\bar{h}(t)-\bar{h}\left(t_{0}\right)\right) d t \\
+\frac{\sum^{\frac{\lfloor N b\rfloor}{N}}}{\int_{k=\lfloor N b\rfloor+\frac{n(N)}{2}-2}^{N(N) \xi_{N}}}\left(\bar{h}(t)-\bar{h}\left(t_{0}\right)\right) d t
\end{array}
$$

where $V_{k, N}=\log \left|U_{k, N}\right|-\mathbb{E}_{\alpha}\left[\log \left|U_{k, N}\right|\right]$. Since $\mathbb{E}_{\alpha}\left[V_{k, N}\right]=0$, using the independence of the random variables $\left(V_{k, N}\right)_{k}$, we get

$$
\begin{aligned}
\operatorname{var}_{\alpha}\left(I_{a, b}\right) & =\frac{1}{n(N) \xi_{N}^{2}} \sum_{k=\lfloor N a\rfloor+1}^{\lfloor N a\rfloor+\frac{n(N)}{2}-1} \sigma^{2}\left(\alpha\left(\frac{k}{N}\right)\right)\left(\int_{\frac{\lfloor N a\rfloor+1}{N}}^{\frac{k+1+\frac{n(N)}{2}}{N}}\left(\bar{h}(t)-\bar{h}\left(t_{0}\right)\right) d t\right)^{2} \\
& +\frac{1}{n(N) \xi_{N}^{2}} \sum_{k=\lfloor N a\rfloor+\frac{n(N)}{2}}^{\lfloor N b\rfloor-\frac{n(N)}{2}-1} \sigma^{2}\left(\alpha\left(\frac{k}{N}\right)\right)\left(\int_{\frac{k+1+\frac{n(N)}{2}}{N}}^{\frac{k+1-\frac{n(N)}{2}}{N}}\left(\bar{h}(t)-\bar{h}\left(t_{0}\right)\right) d t\right)^{2} \\
& \left.+\frac{1}{n(N) \xi_{N}^{2}} \sum_{k=\lfloor N b\rfloor-\frac{n(N)}{2}}^{\frac{\lfloor N b\rfloor}{N}} \sigma^{2}\left(\alpha\left(\frac{k}{N}\right)\right)\left(\int_{\frac{k+1-\frac{n(N)}{2}}{N}}^{\lfloor N b\rfloor+\frac{n(N)}{2}-2}(t)-\bar{h}\left(t_{0}\right)\right) d t\right)^{2} .
\end{aligned}
$$


The two functions $\sigma^{2}$ and $\bar{h}$ are uniformly bounded so it is easy to obtain

$$
\begin{aligned}
\operatorname{var}_{\alpha}\left(I_{a, b}\right) & \leq \frac{K}{N^{2} n(N) \xi_{N}^{2}} \sum_{k=\lfloor N a\rfloor+1}^{\lfloor N a\rfloor+\frac{n(N)}{2}-1}\left(k+\frac{n(N)}{2}-\lfloor N a\rfloor\right)^{2} \\
& +\frac{K}{N^{2} n(N) \xi_{N}^{2}} \sum_{k=\lfloor N a\rfloor+\frac{n(N)}{2}}^{\lfloor N b\rfloor-\frac{n(N)}{2}-1} n(N)^{2} \\
& +\frac{K}{N^{2} n(N) \xi_{N}^{2}} \sum_{k=\lfloor N b\rfloor-\frac{n(N)}{2}}^{\lfloor N b\rfloor+\frac{n(N)}{2}-2}\left(\lfloor N b\rfloor+\frac{n(N)}{2}-1-k\right)^{2} \\
& \leq \frac{K}{N^{2} n(N) \xi_{N}^{2}}\left(n(N)^{3}+([N b]-[N a]) n(N)^{2}+n(N)^{3}\right) \\
& \leq \frac{K^{*}}{\xi_{N}^{2}} \frac{n(N)}{N},
\end{aligned}
$$

whereby

$$
\sup _{\alpha \in \Theta_{1}} \mathbb{E}_{\alpha}\left[\left|\int_{\frac{\lfloor N a\rfloor+1}{N}}^{\frac{\lfloor N b\rfloor}{N}}\left(\hat{h}_{N}^{U}(t)-\bar{h}(t)\right)\left(\bar{h}(t)-\bar{h}\left(t_{0}\right)\right) d t\right|\right] \leq \frac{K^{*}}{\xi_{N}} \sqrt{\frac{n(N)}{N}} .
$$

Gathering (59), (60) and (61), we finally obtain

$$
\mathbb{E}_{\alpha}\left[\left|Q_{5}\right|\right] \leq \frac{K^{*}}{\xi_{N}} \sqrt{\frac{n(N)}{N}} .
$$

Upper bound for $Q_{6}$ The Hölder inequality leads to

$$
\begin{aligned}
\mathbb{E}_{\alpha}\left[\left|Q_{6}\right|\right] & \left.\leq \frac{2}{b-a} \int_{a}^{b} \mathbb{E}_{\alpha}\left[\left|\bar{h}\left(t_{0}\right)-\hat{h}_{N}^{U}\left(t_{0}\right)\right| \mid \hat{h}_{N}^{U}(t)-\bar{h}(t)\right) \mid\right] d t \\
& \left.\leq \frac{2}{b-a} \int_{a}^{b}\left(\left.\mathbb{E}_{\alpha}\left[\left|\bar{h}\left(t_{0}\right)-\hat{h}_{N}^{U}\left(t_{0}\right)\right|^{2}\right] \mathbb{E}_{\alpha}\left[\mid \hat{h}_{N}^{U}(t)-\bar{h}(t)\right)\right|^{2}\right]\right)^{\frac{1}{2}} d t
\end{aligned}
$$

whence

$$
\mathbb{E}_{\alpha}\left[\left|Q_{6}\right|\right] \leq \frac{K}{\xi_{N}^{2}}
$$

thanks to $(25)$.

Upper bound for $Q_{7}$ From the definition (10) of $\bar{h}$, we know that

$$
\left|\frac{1}{b-a} \int_{a}^{b}\left(\bar{h}(t)-\bar{h}\left(t_{0}\right)\right) d t-\frac{1}{b-a} \int_{a}^{b}\left(h(t)-h\left(t_{0}\right)\right) d t\right| \leq K^{*} \frac{n(N)}{N},
$$


whereby Lemma 7 and (50) entail

$$
\begin{aligned}
\mathbb{E}_{\alpha}\left[\left|Q_{7}\right|\right] & \leq K \frac{n(N)}{N \xi_{N}}+\mathbb{E}_{\alpha}\left[\left|\frac{2\left[\bar{h}\left(t_{0}\right)-\hat{h}_{N}^{U}\left(t_{0}\right)\right]}{b-a} \int_{a}^{b}\left(h(t)-h\left(t_{0}\right)\right) d t-\frac{\hat{\sigma}^{2}\left(t_{1}\right)}{\xi_{N}} W_{N}\right|\right] \\
& =K \frac{n(N)}{N \xi_{N}}+\mathbb{E}_{\alpha}\left[\sigma_{\chi}\left|\bar{h}\left(t_{0}\right)-\hat{h}_{N}^{U}\left(t_{0}\right)\right|\left|\frac{\sigma^{2}\left(\alpha\left(t_{1}\right)\right)}{\sqrt{\sigma^{2}\left(\alpha\left(t_{0}\right)\right)}}-\frac{\hat{\sigma}^{2}\left(t_{1}\right)}{\left(B_{N}\right)^{\frac{1}{2}}}\right|\right] .
\end{aligned}
$$

Then we will use the following decomposition:

$$
\frac{\sigma^{2}\left(\alpha\left(t_{1}\right)\right)}{\sqrt{\sigma^{2}\left(\alpha\left(t_{0}\right)\right)}}-\frac{\hat{\sigma}^{2}\left(t_{1}\right)}{\left(B_{N}\right)^{\frac{1}{2}}}=\frac{\sigma^{2}\left(\alpha\left(t_{1}\right)\right)-\hat{\sigma}^{2}\left(t_{1}\right)}{\sqrt{\sigma^{2}\left(\alpha\left(t_{0}\right)\right)}}+\frac{\hat{\sigma}^{2}\left(t_{1}\right)}{\sqrt{\sigma^{2}\left(\alpha\left(t_{0}\right)\right) B_{N}}}\left(\sqrt{B_{N}}-\sqrt{\sigma^{2}\left(\alpha\left(t_{0}\right)\right)}\right) .
$$

Since $\inf _{t} \sigma^{2}(t)>0$, we may choose a constant $K>0$ such that for all $N, B_{N} \geq K$, whence

$$
\left|\frac{\sigma^{2}\left(\alpha\left(t_{1}\right)\right)}{\sqrt{\sigma^{2}\left(\alpha\left(t_{0}\right)\right)}}-\frac{\hat{\sigma}^{2}\left(t_{1}\right)}{\left(B_{N}\right)^{\frac{1}{2}}}\right| \leq K\left|\sigma^{2}\left(\alpha\left(t_{1}\right)\right)-\hat{\sigma}^{2}\left(t_{1}\right)\right|+K^{*}\left|B_{N}-\sigma^{2}\left(\alpha\left(t_{0}\right)\right)\right| .
$$

Then we use the fact that $t \mapsto \sigma^{2}(\alpha(t))$ is a $\mathcal{C}^{1}$ function to obtain a constant $K^{*}>0$ such that $\left|B_{N}-\sigma^{2}\left(\alpha\left(t_{0}\right)\right)\right| \leq K^{*} \frac{n(N)}{N}$. Finally, applying Lemma 7 yields

$$
\mathbb{E}_{\alpha}\left[\left|Q_{7}\right|\right] \leq K^{*} \frac{n(N)}{N \xi_{N}}+K^{*} \mathbb{E}_{\alpha}\left[\left|\bar{h}\left(t_{0}\right)-\hat{h}_{N}^{U}\left(t_{0}\right)\right|\left|\sigma^{2}\left(\frac{1}{h\left(t_{1}\right)}\right)-\sigma^{2}\left(\frac{1}{\hat{h}_{N}\left(t_{1}\right)}\right)\right|\right] .
$$

Consider now a constant $K^{*}>0$ which depends on $\alpha_{*}$ and $\alpha^{*}$ such that

$$
\forall x \in\left[\frac{1}{\alpha^{*}}, \frac{1}{\alpha_{*}}\right] \subset\left(\frac{1}{2}, 1\right), \forall y \in \mathbb{R},\left|\sigma^{2}\left(\frac{1}{x}\right)-\sigma^{2}\left(\frac{1}{y}\right)\right| \leq K^{*}|x-y| .
$$

We have

$$
\left|\sigma^{2}\left(\frac{1}{h\left(t_{1}\right)}\right)-\sigma^{2}\left(\frac{1}{\hat{h}_{N}\left(t_{1}\right)}\right)\right| \leq K^{*}\left|h\left(t_{1}\right)-\hat{h}_{N}\left(t_{1}\right)\right| .
$$

Using the inequality $\left|h\left(t_{1}\right)-\hat{h}_{N}\left(t_{1}\right)\right| \leq\left|h\left(t_{1}\right)-\bar{h}\left(t_{1}\right)\right|+\left|\bar{h}\left(t_{1}\right)-\hat{h}_{N}^{U}\left(t_{1}\right)\right|+\left|\hat{h}_{n}^{U}\left(t_{1}\right)-\hat{h}_{N}\left(t_{1}\right)\right|$, the Hölder inequality and Lemma 7 lead to

$$
\mathbb{E}_{\alpha}\left[\left|Q_{7}\right|\right] \leq K^{*}\left(\frac{n(N)}{N \xi_{N}}+\frac{1}{\xi_{N}^{2}}+\frac{1}{\xi_{N} N^{\left(1-\frac{1}{\alpha_{*}}\right) \frac{\eta}{2}}}\right)
$$

Upper bound for $\mathbb{E}_{\alpha}\left[\left|\chi_{N}-W_{N}\right|\right]$ Putting together all the bounds for $Q_{j}, j=1, \ldots, 7$ we obtain

$$
\sup _{\alpha \in \Theta_{1}} \mathbb{E}_{\alpha}\left[\left|\chi_{N}-W_{N}\right|\right] \leq K_{\eta}^{*}\left[\frac{\xi_{N}}{N^{\left(1-\frac{1}{\alpha_{*}}\right) \frac{\eta}{2}}}+\xi_{N} \frac{n(N)}{N}+\left(\frac{n(N)}{N}\right)^{\frac{1}{2}}+\frac{1}{\xi_{N}}\right]
$$

for some positive constant $K_{\eta}^{*}$. Thanks to (58),

$$
\kappa\left(P_{\chi_{N}}, P_{\chi}\right) \leq K^{*}\left(\frac{1}{\sqrt{n(N)}}+\frac{1}{\sqrt{\sigma_{\chi}}} \sqrt{\left.\frac{\xi_{N}}{N^{\left(1-\frac{1}{\alpha_{*}}\right) \frac{\eta}{2}}}+\xi_{N} \frac{n(N)}{N}+\left(\frac{n(N)}{N}\right)^{\frac{1}{2}}+\frac{1}{\xi_{N}}\right)}\right.
$$


which is (18). Assume now that $n(N)=N^{\gamma}$ with $\gamma \in\left(0, \frac{\alpha_{*}-1}{4 \alpha_{*}}\right)$ in order to prove (19) and (20). Choosing $\eta=\frac{2 \alpha_{*} \gamma}{\alpha_{*}-1}$, (18) becomes

$$
\kappa\left(P_{\chi_{N}}, P_{\chi}\right) \leq K_{\gamma}^{*} \frac{\sqrt{\log N}}{N^{\gamma / 4} \sqrt{\sigma_{\chi}}}
$$

hence (19). Writing $I=\frac{1}{b-a} \int_{a}^{b}\left|h(t)-h\left(t_{0}\right)\right|^{2} d t$, we deduce from the equality $T_{N}=\xi_{N} \chi_{N}+\frac{\xi_{N}^{2}}{\hat{\sigma}^{2}\left(t_{1}\right)} I$ that

$$
\begin{aligned}
\mathbb{P}_{\alpha}\left(T_{N} \geq q_{\beta}\right) & =\mathbb{P}_{\alpha}\left(\chi_{N} \geq \frac{q_{\beta}}{\xi_{N}}-\frac{\xi_{N}}{\hat{\sigma}^{2}\left(t_{1}\right)} I\right)-\mathbb{P}_{\alpha}\left(\chi \geq \frac{q_{\beta}}{\xi_{N}}-\frac{\xi_{N}}{\hat{\sigma}^{2}\left(t_{1}\right)} I\right)+\mathbb{P}_{\alpha}\left(\chi \geq \frac{q_{\beta}}{\xi_{N}}-\frac{\xi_{N}}{\hat{\sigma}^{2}\left(t_{1}\right)} I\right) \\
& \geq \mathbb{P}_{\alpha}\left(\chi \geq \frac{q_{\beta}}{\xi_{N}}-\frac{\xi_{N}}{\hat{\sigma}^{2}\left(t_{1}\right)} I\right)-\kappa\left(P_{\chi_{N}}, P_{\chi}\right) .
\end{aligned}
$$

(20) then follows from (62), noticing that $\sigma^{2} \leq 3$ and that $\chi$ has a symmetric distribution

Remark: If $\frac{1}{b-a} \int_{a}^{b}\left(h(t)-h\left(t_{0}\right)\right) d t=0$ and $\lim _{N \rightarrow+\infty} \frac{n(N)}{N^{\left(1-\frac{1}{\alpha_{*}}\right) \frac{\eta}{2}}}=0$, then $W_{N}=0$. Moreover

$$
\xi_{N}^{2} \mathbb{E}_{\alpha}\left[\left|Q_{1}+Q_{3}+Q_{5}+Q_{6}+Q_{7}\right|\right] \leq K_{\eta}^{*}\left[\frac{\xi_{N}^{2}}{N^{\left(1-\frac{1}{\alpha_{*}}\right) \frac{\eta}{2}}}+\xi_{N}^{2} \frac{n(N)}{N}+\xi_{N}\left(\frac{n(N)}{N}\right)^{\frac{1}{2}}\right]
$$

and $\lim _{N \rightarrow+\infty} \xi_{N}^{2}\left(Q_{1}+Q_{3}+Q_{5}+Q_{6}+Q_{7}\right) \stackrel{\mathbb{P}_{\alpha}}{=} 0$. Furthermore

$$
\lim _{N \rightarrow+\infty} \xi_{N}^{2} Q_{2}=\lim _{N \rightarrow+\infty} \frac{\xi_{N}^{2}}{b-a} \int_{a}^{b}\left|\hat{h}_{N}^{U}(t)-\bar{h}(t)\right|^{2} d t \stackrel{\mathbb{P}_{\alpha}}{=} \frac{1}{b-a} \int_{a}^{b} \sigma^{2}(\alpha(t)) d t
$$

and

$$
\lim _{N \rightarrow+\infty} \xi_{N}^{2} Q_{4}=\lim _{N \rightarrow+\infty} \xi_{N}^{2}\left|\bar{h}\left(t_{0}\right)-\hat{h}_{N}^{U}\left(t_{0}\right)\right|^{2} \stackrel{d}{=} \sigma^{2}\left(\alpha\left(t_{0}\right)\right)|Z|^{2}
$$

where $Z \sim \mathcal{N}(0,1)$. Consequently we have the following convergence in distribution

$$
T_{N}-\frac{\xi_{N}^{2}}{\hat{\sigma}^{2}\left(\alpha\left(t_{1}\right)\right)} \frac{1}{b-a} \int_{a}^{b}\left|h(t)-h\left(t_{0}\right)\right|^{2} d t \stackrel{d}{\longrightarrow} \frac{1}{b-a} \int_{a}^{b} \frac{\sigma^{2}(\alpha(t))}{\sigma^{2}\left(\alpha\left(t_{1}\right)\right)} d t+\frac{\sigma^{2}\left(\alpha\left(t_{0}\right)\right)}{\sigma^{2}\left(\alpha\left(t_{1}\right)\right)} \chi^{2}(1) .
$$

Proof of theorem 3 Recall that $\xi_{N}=\sqrt{n(N)}\left(\Gamma^{\prime}(1)+\log N\right)$ and for $t \in[a, b], W_{N}(t)=$ $\xi_{N}\left(\hat{h}_{N}(t)-h(t)\right)$. From the definition (14) of $T_{N}$, we have

$$
T_{N}=\frac{1}{\hat{\sigma}^{2}\left(t_{1}\right)(b-a)} \int_{a}^{b}\left|W_{N}(t)-W_{N}\left(t_{0}\right)+\xi_{N}\left(h(t)-h\left(t_{0}\right)\right)\right|^{2} d t .
$$


Therefore for any $x>0$ and $\alpha \in \Theta_{1}$, writing $I=\frac{1}{b-a} \int_{a}^{b}\left|h(t)-h\left(t_{0}\right)\right| d t$, we compute

$$
\begin{aligned}
\mathbb{P}_{\alpha}\left(T_{N} \leq x\right) & \leq \mathbb{P}_{\alpha}\left(\frac{1}{b-a} \int_{a}^{b}\left|W_{N}(t)-W_{N}\left(t_{0}\right)+\xi_{N}\left(h(t)-h\left(t_{0}\right)\right)\right| d t \leq\left(x\left\|\sigma^{2}\right\|_{\infty}\right)^{1 / 2}\right) \\
& \leq \mathbb{P}_{\alpha}\left(\xi_{N} I \leq K \sqrt{x}+\frac{1}{b-a} \int_{a}^{b}\left|W_{N}(t)-W_{N}\left(t_{0}\right)\right| d t\right) \\
& =\mathbb{P}_{\alpha}\left(e^{\xi_{N} I} \leq e^{K \sqrt{x}} e^{\frac{1}{b-a} \int_{a}^{b}\left|W_{N}(t)-W_{N}\left(t_{0}\right)\right| d t}\right) \\
& \leq \mathbb{P}_{\alpha}\left(e^{\xi_{N} I} \leq e^{K \sqrt{x}} e^{\left|W_{N}\left(t_{0}\right)\right|} e^{\frac{1}{b-a} \int_{a}^{b}\left|W_{N}(t)\right| d t}\right)
\end{aligned}
$$

The Markov inequality combined with the Hölder inequality yields

$$
\mathbb{P}_{\alpha}\left(T_{N} \leq x\right) \leq e^{K \sqrt{x}} e^{-\xi_{N} I}\left(\mathbb{E}_{\alpha}\left[e^{2\left|W_{N}\left(t_{0}\right)\right|}\right]\right)^{1 / 2}\left(\mathbb{E}_{\alpha}\left[e^{\frac{1}{b-a} \int_{a}^{b} 2\left|W_{N}(t)\right| d t}\right]\right)^{1 / 2}
$$

and the Jensen inequality leads to

$$
\mathbb{P}_{\alpha}\left(T_{N} \leq x\right) \leq e^{K \sqrt{x}} e^{-\xi_{N} I}\left(\mathbb{E}_{\alpha}\left[e^{2\left|W_{N}\left(t_{0}\right)\right|}\right]\right)^{1 / 2}\left(\frac{1}{b-a} \int_{a}^{b} \mathbb{E}_{\alpha}\left[e^{2\left|W_{N}(t)\right|}\right] d t\right)^{1 / 2}
$$

The inequality (21) is finally deduced from (28)

\section{References}

Ayache, A. (2013). "Sharp estimates on the tail behavior of a multistable distribution". In: Statistics and Probability Letters 83.3, pp. 680-688.

Ayache, A. and J. Lévy-Véhel (2000). "The generalized multifractional Brownian motion". In: Stat. Inference Stoch. Process. 3, pp. 7-18.

Beghin, L. and C. Ricciuti (2018). "Time-inhomogeneous fractional Poisson processes defined by the multistable subordinator". In: Stochastic Analysis and Applications. DOI: 10.1080/ 07362994.2018 .1548970$.

Benassi, A., S. Jaffard, and D. Roux (1997). "Gaussian processes and pseudodifferential elliptic operators". In: Rev. Mat. Iberoamericana 13, pp. 19-89.

Biermé, H. and C. Lacaux (2013). "Linear multifractional multistable motion: LePage series representation and modulus of continuity". In: Ann. Univ. Bucharest (Math. Series) 4.LXII, pp. $345-360$.

Biermé, H. and F.J.P. Richard (2010). "Statistical tests of anisotropy for fractional Brownian textures. Application to Full-field Digital Mammography". In: J Math Imaging Vis 36, pp. 227-240. DOI: $10.1007 / \mathrm{s} 10851-009-0181-\mathrm{y}$.

Falconer, K.J. (2002). "Tangent fields and the local structure of random fields". In: J. Theoret. Probab. 15, pp. 731-750.

Falconer, K.J. (2003). "The local structure of random processes". In: J. London Math. Soc. 67.2, pp. $657-672$.

Falconer, K.J. and J. Lévy-Véhel (2008). "Multifractional, multistable, and other processes with prescribed local form". In: J. Theoret. Probab. DOI: 10.1007/s10959-008-0147-9. 
Falconer, K.J. and L. Liu (2012). "Multistable Processes and Localisability". In: Stochastic Models 28, pp. 503-526.

Ferguson, T.S. and M.J. Klass (1972). "A representation of independent increment processes without Gaussian components". In: Ann. Math. Stat. 43, pp. 1634-1643.

Herbin, E. (2006). "From N-parameter fractional Brownian motions to N-parameter multifractional Brownian motion". In: Rocky Mountain J. Math. 36, pp. 1249-1284.

Le Guével, R. (2013). "An estimation of the stability and the localisability functions of multistable processes". In: Electronic Journal of Statistics 7, pp. 1129-1166. DOI: 10.1214/13EJS797.

Le Guével, R. (2018). "The Hausdorff dimension of the range of the Lévy multistable processes". In: Journal of Theoretical Probability. DOI: 10.1007/s10959-018-0847-8.

Le Guével, R. and J. Lévy-Véhel (2012). "A Ferguson-Klass-LePage representation of multistable multifractional processes and related processes". In: Bernoulli 18.4, pp. 1099-1127. DOI: $10.3150 . / / 11-B E J 372$.

Le Guével, R. and J. Lévy-Véhel (2013). "Incremental moments and Hölder exponents of multifractional multistable processes". In: ESAIM:PS. DOI: http://dx.doi.org/10.1051/ps/ 2011151.

Le Guével, R., J. Lévy-Véhel, and L. Liu (2012). “On two multistable extensions of stable Lévy motion and their semimartingale representation". In: J. Theoret. Proba. DOI: 10.1007/ s10959-013-0528-6.

Loeve, M. (1977). Probability Theory I (4th edn). Springer.

Molchanov, I. and K. Ralchenko (2015). "Multifractional Poisson process, multistable subordinator and related limit theorems". In: Stat. Probab. Lett. 96, pp. 95-101.

Orsingher, E., C. Ricciuti, and B. Toaldo (2016). "Time-inhomogeneous jump processes and variable order operators". In: Potential Anal 45, pp. 435-461. DOI: 10.1007/s11118-0169551-4.

Peltier, R.F. and J. Lévy-Véhel (1995). "Multifractional Brownian motion: definition and preliminary results". In: Rapport de recherche de l'INRIA. URL: http: / / www-rocq1 . inria . $\mathrm{fr} /$ fractales/index. php?page=publications.

Petrov, V. (1995). Limit Theorems of Probability Theory. Oxford Science Publication.

Ricciuti, C. and B. Toaldo (2017). "Semi-Markov models and motion in heterogeneous media". In: J. Stat. Phys. 45, pp. 169-340. URL: https://doi.org/10.1007/s10955-017-1871-2.

Richard, F.J.P. and H.T.L. Vu (2019). "Statistical tests of heterogeneity for anisotropic multifractional Brownian fields". In: Working paper or preprint. URL: https://hal . archivesouvertes.fr/hal-01863377.

Rosinski, J. (1990). "On Series Representations of Infinitely Divisible Random Vectors". In: Ann. Probab. 18.1, pp. 405-430.

Samorodnitsky, G. and M.S. Taqqu (1994). Stable Non-Gaussian Random Processes. Chapman and Hall.

Stoev, S. and M.S. Taqqu (2004). "Stochastic properties of the linear multifractional stable motion". In: Adv. Appl. Probab. 36, pp. 1085-1115.

Stoev, S. and M.S. Taqqu (2005). "Path properties of the linear multifractional stable motion". In: Fractals 13, pp. 157-178.

Von Bahr, B. and C.G. Essen (1965). "Inequalities for the rth Absolute Moment of a Sum of Random Variables, $1<=\mathrm{r}<=2$ ". In: The Annals of Mathematical Statistics 36.1, pp. 299303. 